RICE UNIVERSITY

\title{
Suspended Si Ring Resonator for Mid-IR Application
}

\author{
by \\ Yang Xia
}

\author{
A Thesis Submitted \\ in Partial Fulfillment of the \\ ReQUiRements For THE Degree \\ Master of Science
}

Approved, Thesis CommitTeE:

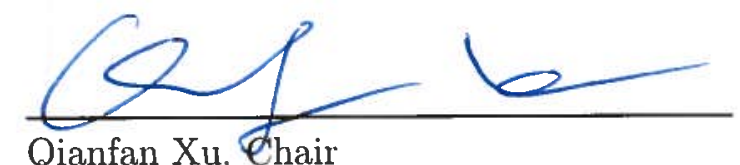

Qianfan Xu, ehair

Assistant Professor of Electrical and Computer Engineering-

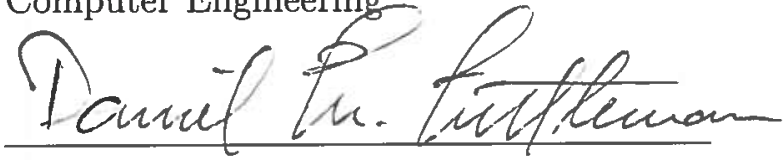

Daniel M. Mittleman

Professor of Electrical and Computer Engineering

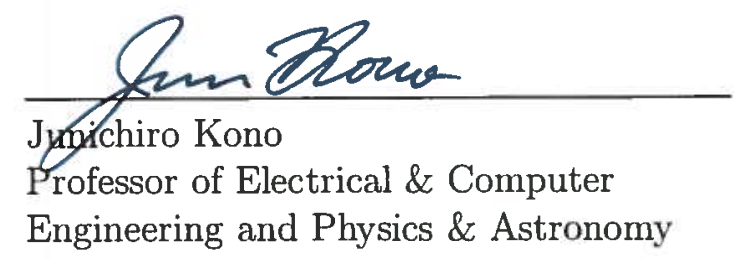

Houston, Texas

December, 2013 


\section{ABSTRACT \\ Suspended Si Ring Resonator for Mid-IR Application \\ by \\ Yang Xia}

Suspended ring resonators formed by both single-mode waveguide (SMW) and multi-mode waveguide (MMW) are designed, fabricated and characterized near $3.4 \mu \mathrm{m}$ by thermal tuning and near $4.5 \mu \mathrm{m}$ and $5.2 \mu \mathrm{m}$ by tunable quantum cascade lasers. The dispersion property is analyzed by simulation in regards to frequency comb generation. The taper width is optimized for maximum coupling. Measurement setup is built up and described. For the SMW ring resonator, the intrinsic quality factor is fitted to be 6,800 and 16,000 near $5.2 \mu \mathrm{m}$ and $4.5 \mu \mathrm{m}$, respectively. For the MMW ring resonator, it rises to 35,000 near $4.5 \mu \mathrm{m}$. Transmission spectrum distortion is observed at high input power, and is modeled as heat effect. Thermal tuning rate is experimentally confirmed at $0.21 \mathrm{~nm} /{ }^{\circ} \mathrm{C}$. Based on the measured distortion and heat simulation, absorption loss is estimated. Alloptical modulation is conducted to estimate the response time of this process. It can be shown that main loss is from surface thus is reducible by improving surface quality. On-chip electrical heater is designed and preliminary experiment indicate the feasibility to pattern it with our Electron Beam Lithography system. 


\section{Acknowledgments}

First of all, I would like to thank my adviser Qianfan Xu for his patience and kindness to me, and for the hands-on demonstration and inspiring discussion. Besides, I would like to thank Ciyuan Qiu for his detailed instructions on U-shape waveguide device design, electron beam lithography, measurement system build-up and device measurement. Last but not the least I would like to thank Weilu Gao and Jie Shu for their helpful discussion about fabrication techniques. 


\section{Content}

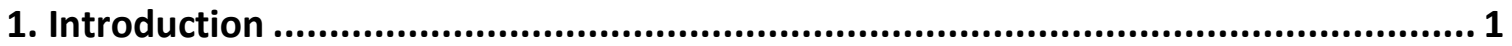

2. Device design and fabrication ...............................................................................6

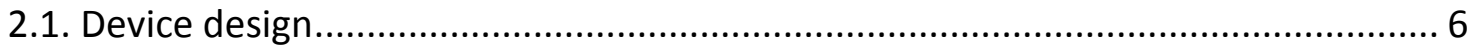

2.1.1. The concept of the suspended waveguide on SOI wafer ............................... 6

2.1.2. Device layout ..................................................................................................... 7

2.1.3. Dimensions of the waveguide and dispersion property................................. 8

2.2. The fabrication process .......................................................................... 12

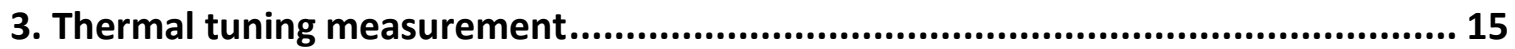

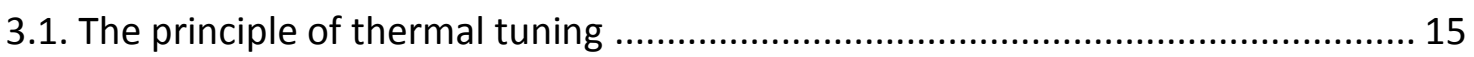

3.2. Measurement setup for mid-IR U-shape waveguide .......................................... 16

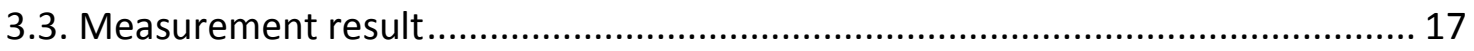

4. Direct spectrum measurement by tunable QCL …................................................. 19

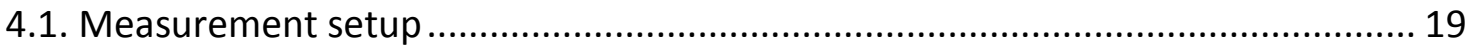

4.2. Transmission spectra of the SMW resonator..................................................... 19

4.3. Transmission spectra of MMW resonator ............................................................ 23

4.4. Thermal tuning rate measurement ............................................................... 25

5. All-optical modulation ............................................................................................. 26

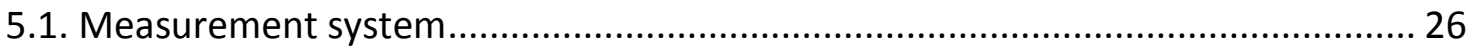

5.2. All-optical modulation results .......................................................................... 28

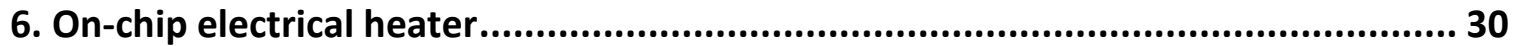

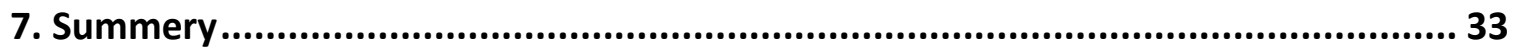

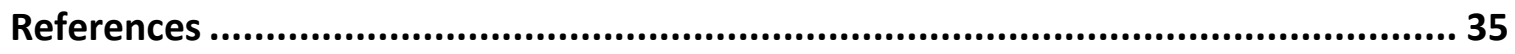




\section{List of Figures}

Fig. 1 The spectrum fingerprints of some common gases [8] ............................. 2

Fig. 2 The MIR transparency window (waveguide propagation loss $\leqslant 2 \mathrm{~dB} / \mathrm{cm}$ ) for diffrerent materials. The white areas indicate the transparent window; red areas present high loss. [9]

Fig. 3 Frequency comb generated in microresonator by Kerr nonlinearity. The comb spectra are generated by (A) silica microntoroidal resonator, (B) $\mathrm{CaF}_{2}$ crystaline resonator, (C) integrate SiN resonator. The photos of different resonators are shown in (D). [5].

Fig. 4 The structure of the suspended silicon waveguide (a) [20] and simulated mode distribution for the $1.5 \mu \mathrm{m}$ wide waveguide (b) 7

Fig. 5 The SEM photos for a whole device (a) and for an individule ring resonator (b)[20] 8

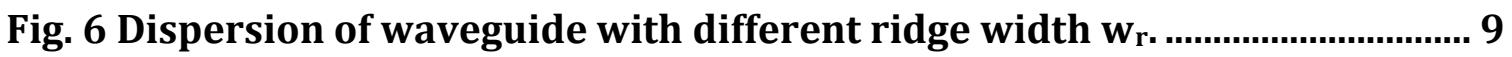

Fig. 7 Simulated taper tip coupling efficiency for different tip width. 11

Fig. 8 Fabrication procedures for the suspended Si MIR waveguide and resonator. The order is from left to right, from up to dowe.

Fig. 9 The optimization of the RIE recipe to realize vertical side wall. (a) The etching result of the original recipe $\left(\mathrm{SF}_{6} 30 / \mathrm{O}_{2} 10 / \mathrm{CHF}_{3} 12\right)$ [24]; (b) The etching result of the improved recipe $\left(\mathrm{SF}_{6} 30 / \mathrm{O}_{2} 10 / \mathrm{CHF}_{3} 7\right)$; (c) The etching result of the final recipe $\left(\mathrm{SF}_{6} 30 / \mathrm{O}_{2} 14 / \mathrm{CHF}_{3} 12\right)$.

Fig. 10 The microscope photos of the device after (a) patterned resist for the waveguide and ring (b) transfering the pattern of waveguide and rings to the substrate by RIE (c) opening the holes on the substrate by RIE and (d) suspending the waveguide and ring. 14

Fig. 11 Thermal tuning measuremnt system........................................................... 17

Fig. 12 A typical thermal tuning spectrum [20]

Fig. 13 Normalized transmission spectra of a narrow-ridge ring resonator [20] 
Fig. 14 The COMSOL model for the simulation. Axial symmetry condition is applied on the left edge. The temperature of all four edges is fixed at $295 \mathrm{~K}$. The initial temperature of all parts is also $295 \mathrm{~K}$. The heating is assumed to be homogenouse at the ridge area. The ridge is $1.5 \mu \mathrm{m}$ wide. The height of the ridge and the thickness of the slab are $0.5 \mu \mathrm{m}$ and $0.25 \mu \mathrm{m}$ respectively........22

Fig. 15 Transmission spectrum of the SMW resonator near $4.5 \mu \mathrm{m} . . . \ldots \ldots \ldots \ldots \ldots . . . . .23$

Fig. 16 The transmission spectrum and fitting result for a wide-ridge resonator. 24

Fig. 17 Thermal tuning rate measurement. The green squares are measured at $22{ }^{\circ} \mathrm{C}$ and fitted in the blue curve, while the orange squares are measured at $27^{\circ} \mathrm{C}$ and fitted in the red curve. 25

Fig. 18 All-optical thermo-optical modulation measurment system..................27

Fig. 19 The principle of all-optical thermal-optical modulation..........................27

Fig. 20 Optical modulation response with respect to pump wavelength...........28

Fig. 21 Bandwidth measurement of the thermo-optical modulation [20]........29

Fig. 22 On-chip electrical heater design. The heater and electrodes are shown as the area surrounded by red curves.

Fig. 23 Lithography result of the heater. 


\section{Chapter 1}

\section{Introduction}

Mid-infrared (MIR) wavelength range $(2-20 \mu \mathrm{m})$ holds important position in scientific research and industrial application. Many molecules have spectrum fingerprint in this range, thus can be distinguished and studied with MIR spectroscopy. [1, 2] It also contains two relative transparent windows of the atmosphere. Unfortunately, this is also a wavelength range where currently flourishing integrated photonics platform silicon-on-insulator (SOI) wafer is not appropriate, because the $\mathrm{SiO}_{2}$ under the waveguide structure has strong absorption beyond this wavelength. [3] As an alternative, waveguides fabricated with silicon on sapphire (SOS) wafer has been exploited. [4, 5] But such kind of wafer is less commonly used. To overcome the problem of SOI platform, suspended waveguide structure was proposed and demonstrated. $[3,6,7]$ 


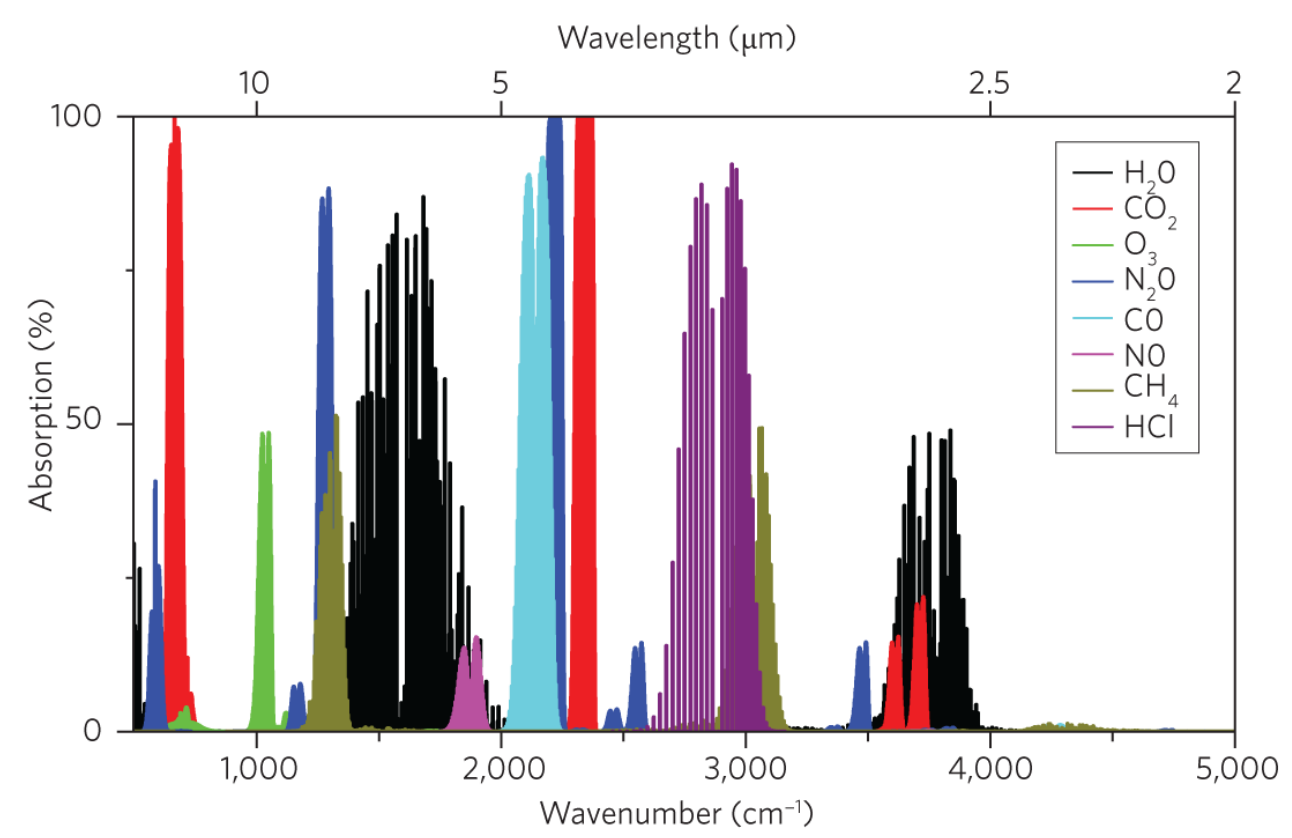

Fig. 1 The spectrum fingerprints of some common gases [8]

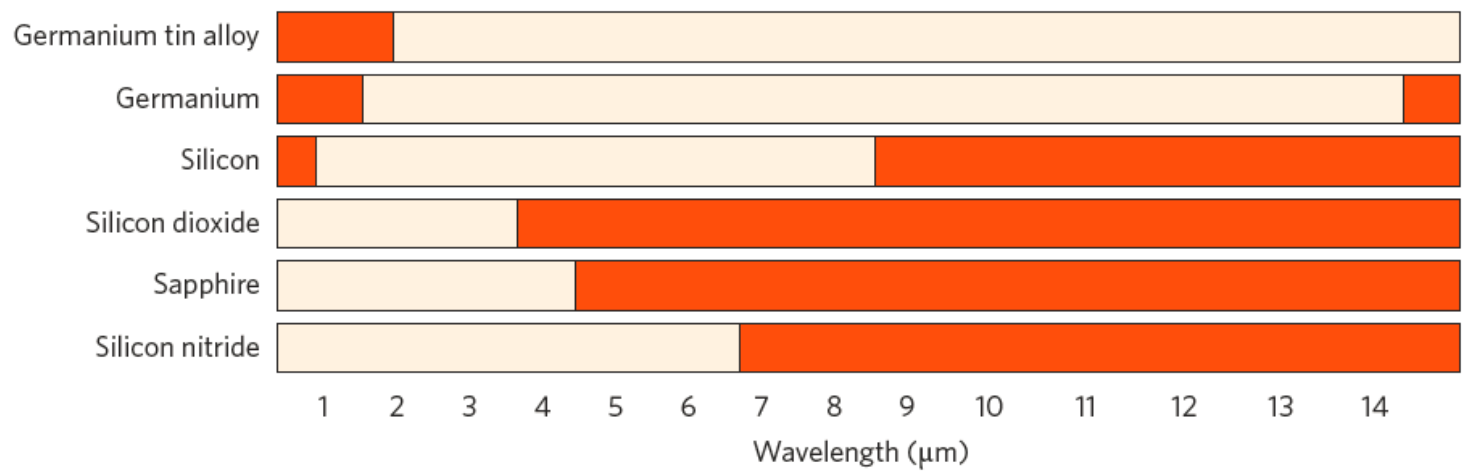

Fig. 2 The MIR transparency window (waveguide propagation loss $\leq 2 \mathrm{~dB} / \mathrm{cm}$ ) for diffrerent materials. The white areas indicate the transparent window; red areas present high loss. [9] 
An especially interesting topic for Mid-IR silicon photonics is optical nonlinearity. Silicon has large linear and nonlinear permittivity. [10] The former guarantees strong optical confinement, while the latter provides large nonlinear response. However, silicon suffers from two photon absorption (TPA) and free carrier absorption (FCA) when working in telecom wavelength $(\sim 1.5 \mu \mathrm{m})$. This limitation disappears when the working wavelength moves beyond $2.2 \mu \mathrm{m}$. [10] On the other hand, highly developed silicon processing techniques enables high device fabrication quality. Thus it is quite promising for nonlinear application in Mid-IR range. Previous research mainly focuses on wavelength conversion based on fourwave mixing in silicon waveguide. [10-12] However, the $\mathrm{SiO}_{2}$ cladding is not removed in those works, thus the working wavelength is limited within the transparent window of $\mathrm{SiO}_{2}$.

Laser frequency combs have been one of the most versatile tools in modern light wave technology, since their introduction in the late 1990s. This concept is inspired from the spectrum generated by mode-locked femtosecond laser, in which millions of laser modes coherently oscillating with a common frequency spacing equal to the repetition rate of the pulse train. It has profound application in optical clock [13], frequency calibration [14, 15] and spectroscopy [16]. In the 2000s, frequency comb was generated in optical microresonators by cascaded four wave mixing (FWM). [17] Since FWM is originated from Kerr effect, such kind of frequency combs are also called Kerr combs. Kerr combs have been generated in silica microtoroid resonator [17], crystalline resonator [18] and integrated SiN 
resonator [19]. Although silicon is not desirable for a comb in telecom wavelength (1.3 - $1.5 \mu \mathrm{m})$, it should make excellent frequency comb generator in MIR.
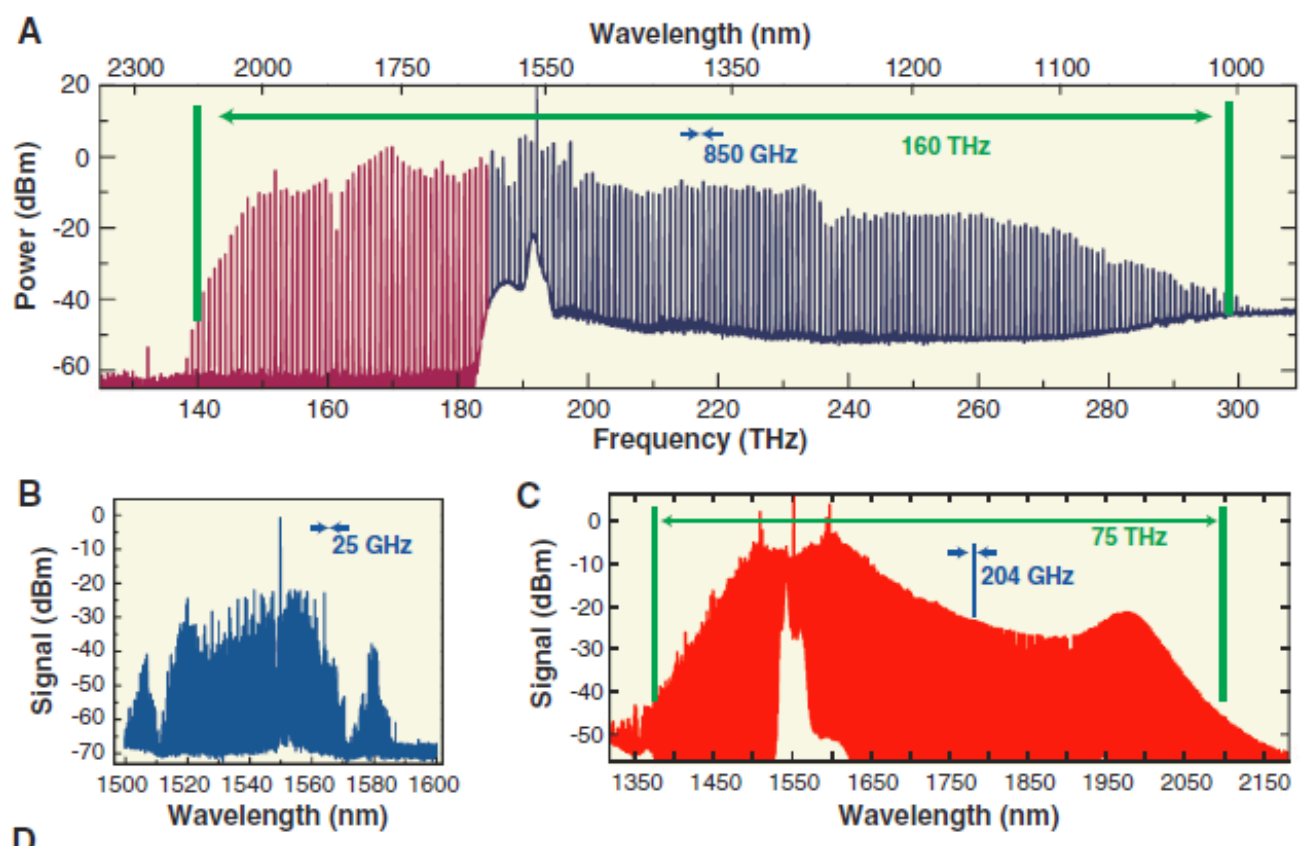

D

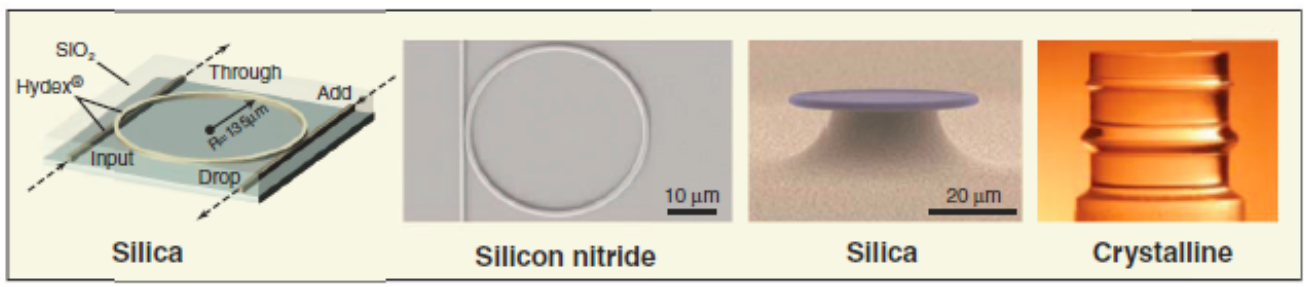

Fig. 3 Frequency comb generated in microresonator by Kerr nonlinearity. The comb spectra are generated by $(A)$ silica microntoroidal resonator, $(B) \mathrm{CaF}_{2}$ crystaline resonator, (C) integrate SiN resonator. The photos of different resonators are shown in (D). [5]

Here we will demonstrate the suspended silicon waveguide and ring resonator as a first step towards an on-chip MIR frequency comb spectroscopy platform. [20] The design consideration and fabrication procedures are presented. The dispersion property is analyzed from simulation result. We describe the 
thermal tuning measurement principle, system setup and transmission spectrum measured at $3.4 \mu \mathrm{m}$ by this principle. Direct transmission spectrum measurement is also done with tunable quantum cascade lasers (QCLs) at $5.2 \mu \mathrm{m}$ and $4.5 \mu \mathrm{m}$. Spectrum distortion due to thermal effect is observed and analyzed. The thermal effect is further verified by all-optical modulation, where the signal light at $3.4 \mu \mathrm{m}$ is modulated by pulsed pump light at $5.2 \mu \mathrm{m}$. Simulation result predicts comparable bandwidth as the experimental result. Finally, the design and preliminary experiment are presented about on-chip electrical heater. 


\section{Chapter 2}

\section{Device design and fabrication}

\subsection{Device design}

\subsubsection{The concept of the suspended waveguide on SOI wafer}

The basic suspended Si waveguide for mid-IR application was proposed in [21]. The structure is fabricated on SOI wafer. A ridge waveguide is made on the top Si layer. The buried oxide (BOX) is removed locally under the ridge area, resulting in an air-cladding waveguide structure. An experimental demonstration was achieved in [6]. In our design, holes are open along the both sides of the ridge on the silicon slab, with proper distance between the holes and the ridge, thus buffered oxide etch can reach and remove the oxide underneath. By controlling the etching time, the width of the suspended area is determined. However, the etching time should be long enough to make the etching holes join together and remove the oxide in the mode area completely. 

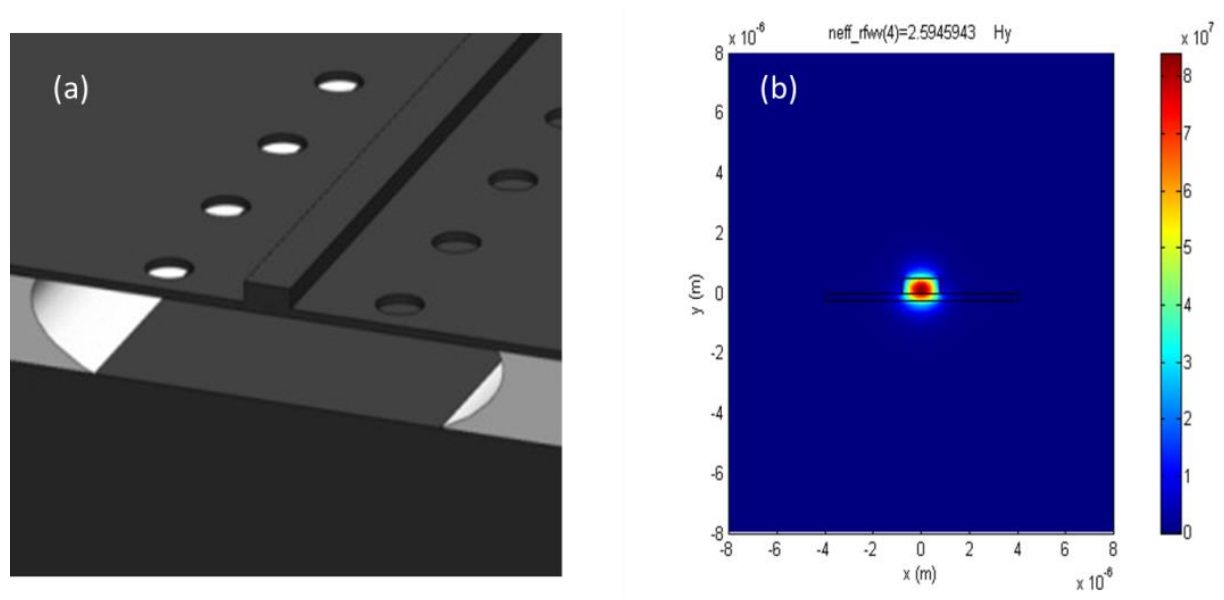

Fig. 4 The structure of the suspended silicon waveguide (a) [20] and simulated mode distribution for the $1.5 \mu \mathrm{m}$ wide waveguide (b)

\subsubsection{Device layout}

Multiple devices are fabricated in a row on one edge of a chip. In each device, there are several rings, as shown in Fig. 5. Each one is designed with a slightly different diameter in such a way that their resonance wavelengths of the same order distribute evenly in one FSR. [20] They are all side coupled to a U-shape waveguide which is ended at the same edge of the chip. [20,22] The ends of the U-shape waveguide are tapered to increase the coupling between the free space optics and the guided wave mode. 

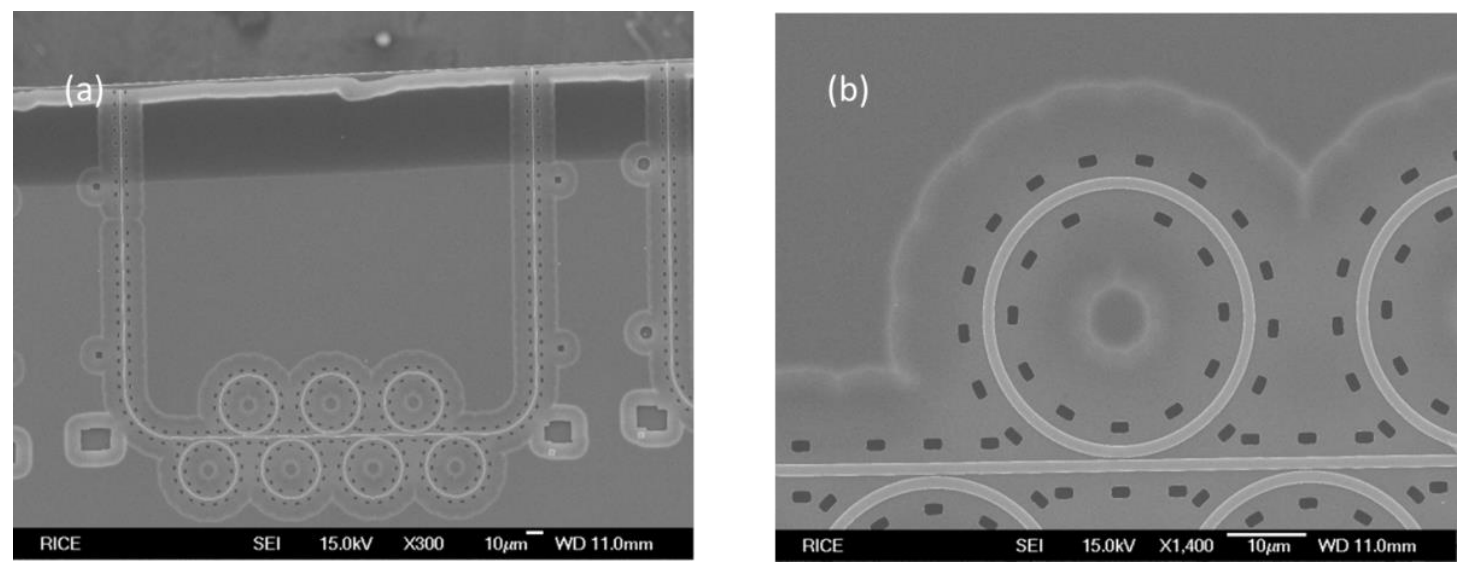

Fig. 5 The SEM photos for a whole device (a) and for an individule ring resonator (b)[20]

\subsubsection{Dimensions of the waveguide and dispersion property}

To maximize comb generation efficiency, waveguide dispersion should be minimized, while keep the mode volume not too large. The FWM process requires both momentum $\left(k_{i}+k_{s}=k_{p 1}+k_{p 2}\right)$ and energy conservation $\left(\omega_{i}+\omega_{s}=\omega_{p 1}+\omega_{p 2}\right)$. For the cavity modes, momentum conservation is naturally satisfied since the propagation constant for the m-order mode is determined by $2 \pi R k_{m}=2 m \pi$, where $R$ is the diameter of the resonator. [18] The energy conservation, on the other hand, is not easy to satisfy, given the group velocity dispersion GVD is generously not zero. The result is non-evenly spacing resonant frequencies, which gives low conversion efficiency.

To accommodate this requirement, the height of the ridge and the thickness of the slab are designed to be $0.5 \mu \mathrm{m}$ and $0.25 \mu \mathrm{m}$. We have two widths for the ridge. The narrow one is $1.5 \mu \mathrm{m}$, and is close to the widest dimension for single mode 
operation, (referred as SMW below). The wider one is $3 \mu \mathrm{m}$ wide (referred as MMW below), and provides lower and dispersion (see Fig. 6). [5] The thickness of BOE is 3 $\mu \mathrm{m}$. The diameter of resonator is $\sim 35 \mu \mathrm{m}$ for the SMW resonator and $\sim 60 \mu \mathrm{m}$ for the MMW resonator. The distance between the side walls of the holes and the ridge is larger than $2 \mu \mathrm{m}$. From simulation, the electromagnetic field within this width contain $>99.5 \%$ of total transmission power even for the narrow waveguide and at 6 $\mu \mathrm{m}$ wavelength, except for the taper coupling area, where the mode is wider since the waveguide is narrower. Thus the holes have only negligible perturbation to the waveguide mode.

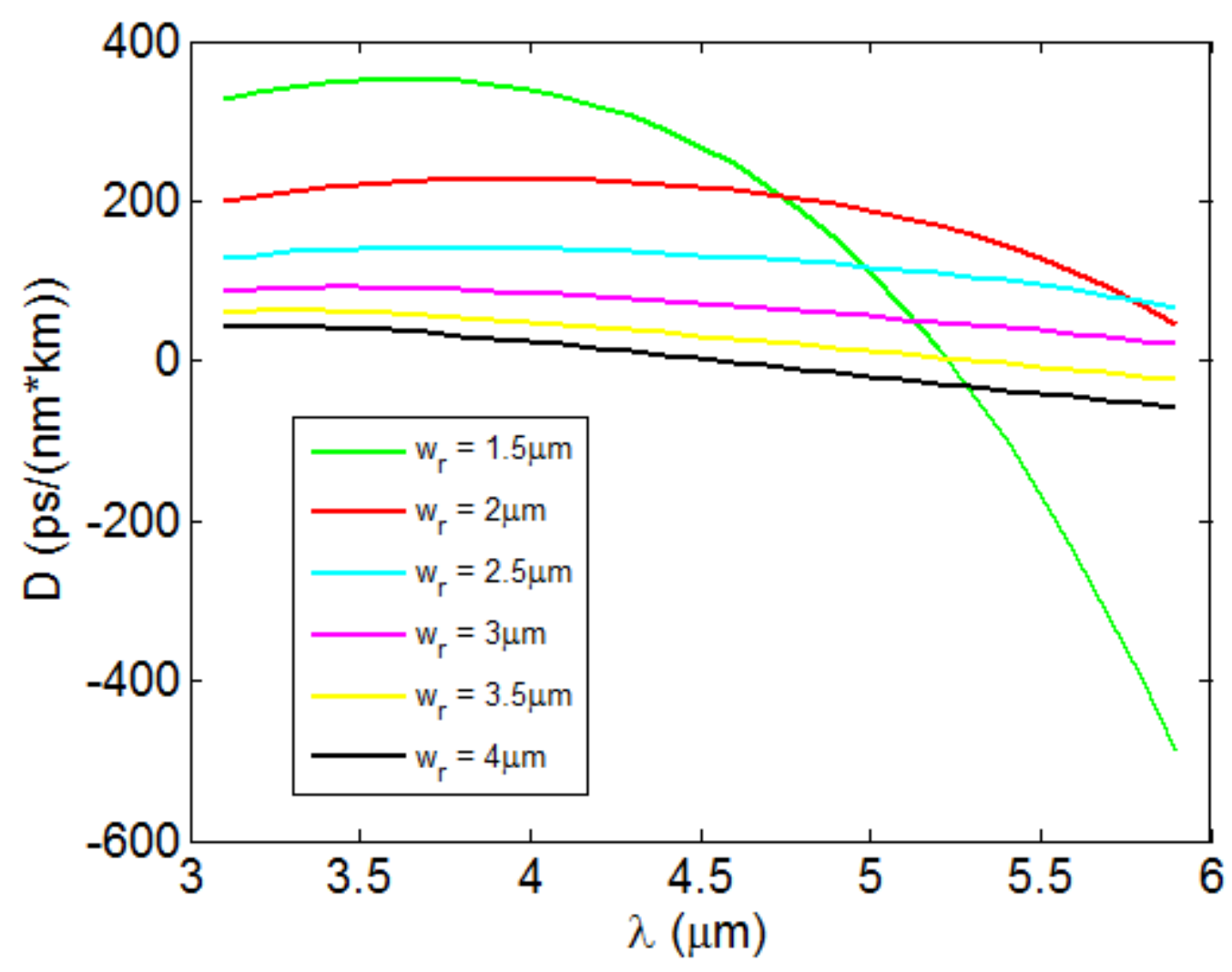

Fig. 6 Dispersion of waveguide with different ridge width $w_{r}$. 
Based on [23], the significance of the dispersion is indicated by the absolute value of the ratio $\Psi=2 \triangle F S R / F W H M$, where $\triangle F S R$ is the difference between two successive free space range (FSR), while FWHM is the full width at half maximum of the resonance in the cavity. When $|\Psi| \geq 1$ multiple harmonic parametric process will not exist in the cavity. And the dispersion can be considered small only when $\Psi \ll 1$. For our MMW resonator with $60 \mu \mathrm{m}$ diameter, the group velocity $\mathrm{n}_{\mathrm{g}}$ and dispersion $\mathrm{D}$ are estimated at 3.7 and $72 \mathrm{ps} / \mathrm{km} \cdot \mathrm{nm}$ at $4.5 \mu \mathrm{m}$ wavelength respectively, which gives $\triangle \mathrm{FSR}=-73 \mathrm{MHz}$. Consider a ring with loaded $\mathrm{Q} \sim 20,000$ (which is the case we achieved in this thesis), we have $\Psi \sim 0.04$, which indicates a small dispersion. However, when we further enhance the $\mathrm{Q}$ value, smaller $\triangle \mathrm{FSR}$ may be necessary, which can be obtained by either using a smaller FSR or smaller dispersion (D or $\beta_{2}$ ).

To optimize the dimension of the taper, we simulate the coupling between the guided mode and a Gaussian beam. The diameter of the Gaussian beam is set to be $3.5 \mu \mathrm{m}$, which is calculated from the divergence angle and the focus length of the coupling lens. This number is also close to experimental value estimated from the light spot from the image. The major insertion loss occurs at the tip of the taper due to the large mode mismatching. Thus we just need to optimize the width of the ridge at the tip to maximize the coupling at this point. It turns out that the width of $0.4 \mu \mathrm{m}$ gives the best coupling efficiency $\sim 43 \%$, corresponding to an insertion loss of 3.7 $\mathrm{dB}$ for each interface. The best experiment result is $\sim 5 \mathrm{~dB}$. 


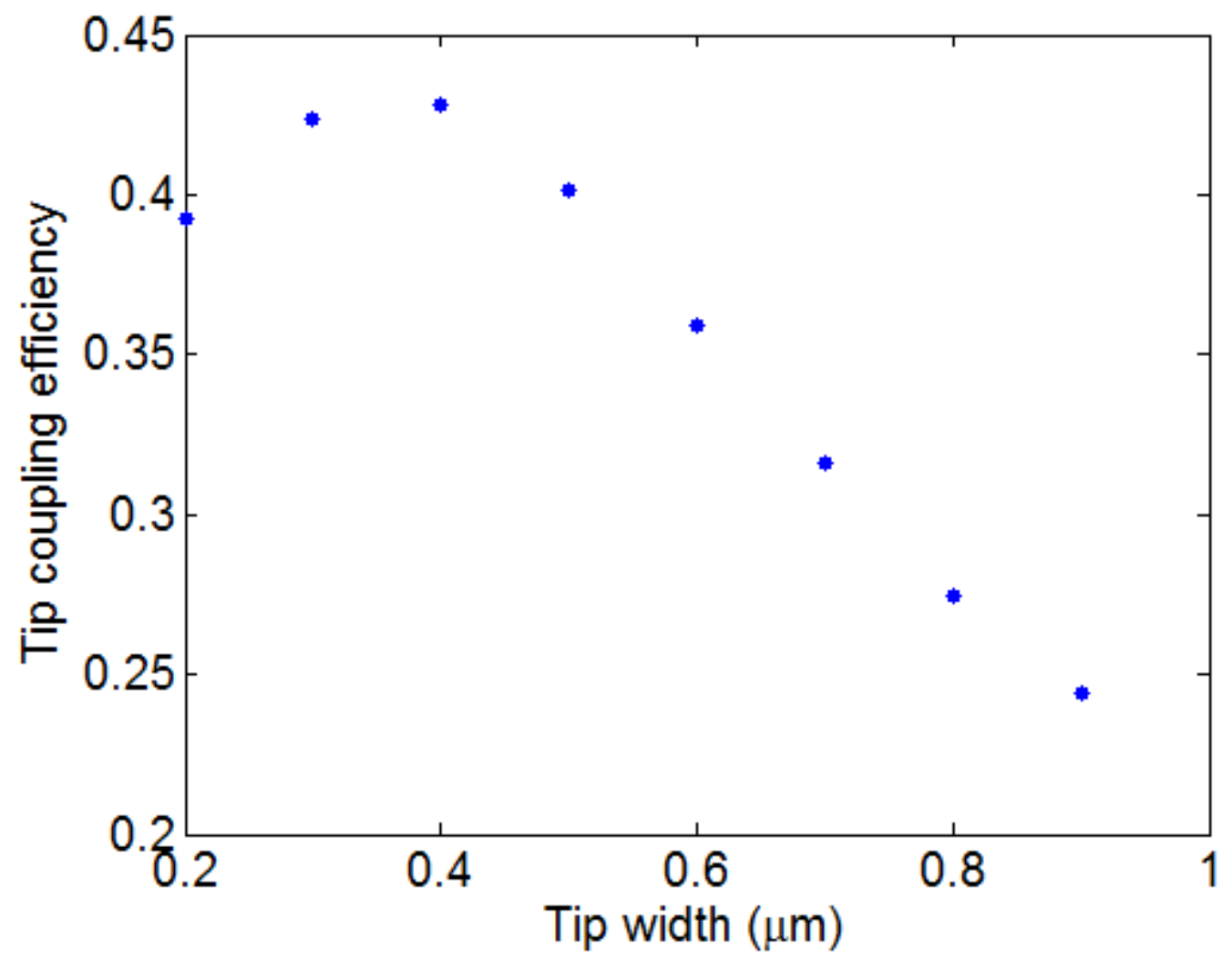

Fig. 7 Simulated taper tip coupling efficiency for different tip width. 


\subsection{The fabrication process}

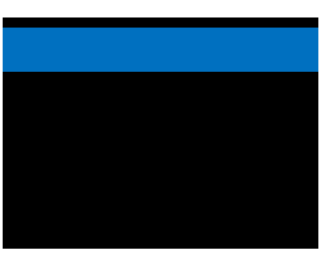

Original SOI wafer

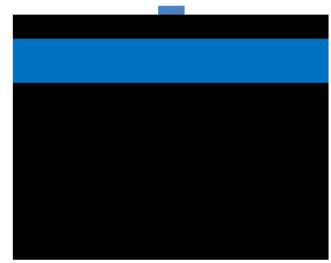

Pattern the resist with electron beam lithography (EBL) to make waveguides

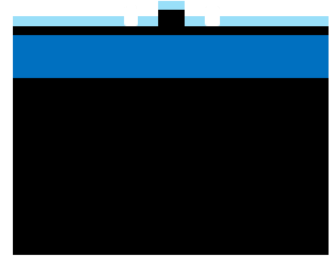

Pattern the resist with EBL to make holes

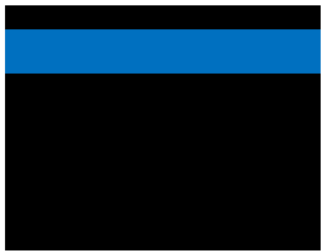

Epitaxy grow the top Si layer from $0.25 \mu \mathrm{m}$ to $0.75 \mu \mathrm{m}$

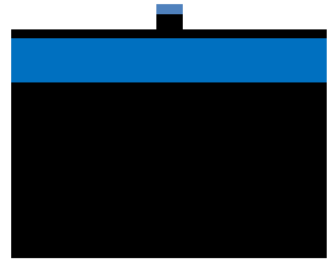

Transfer the pattern to substrate with RIE

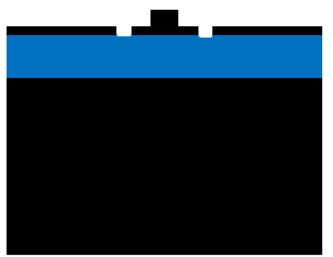

Transfer the pattern to substrate with RIE

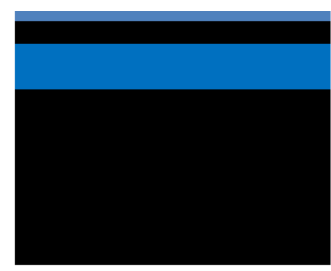

Spin negative resist XR1541 on the wafer

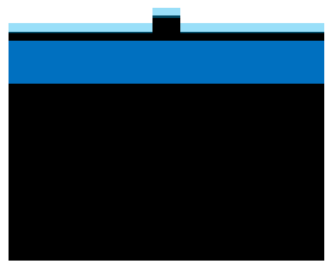

Spin positive resist PMMA on the wafer

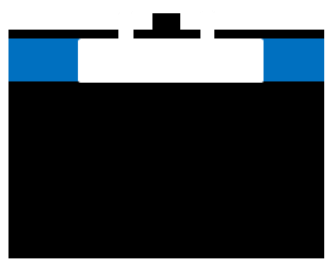

Suspend the waveguide with buffered $\mathrm{HF}$

Fig. 8 Fabrication procedures for the suspended Si MIR waveguide and resonator. The order is from left to right, from up to dowe.

The fabrication process is shown as Fig. 8. It starts from thickening the top layer of the SOI wafer from $0.25 \mu \mathrm{m}$ to $0.75 \mu \mathrm{m}$ by epitaxy growing. [20] The ridge is fabricated on the thickened top layer, followed by opening the holes and removing the BOX. A layer of negative resist for electron beam lithography XR1541 is spun on the top of wafer at the speed of $1000 \mathrm{rpm}$ for $45 \mathrm{~s}$. Then the pattern of the ridge 
waveguide is defined on it by electron beam lithography (EBL), and transferred to the silicon layer by reactive ion etch (RIE), with an optimized $\mathrm{CF}_{4} / \mathrm{CHF}_{3} / \mathrm{O}_{2}$ recipe (see Fig. 9). The height of the ridge is controlled by the etching time. Afterwards, a layer of PMMA A3 is spun on the slab at the speed of $1500 \mathrm{rpm}$ for $45 \mathrm{~s}$. The pattern of holes is defined on the PMMA resist by EBL, and transferred to the slab by RIE. To guarantee the holes are opened completely, over etch is necessary. When using the recipe containing $\mathrm{O}_{2}$, a passivation layer is usually formed at the surface of the etched area, [24] which stops the invading of BOE. We found that oxygen plasma can remove this layer effectively. After this treatment, the device is immerged into 5:1 BHF to locally remove BOX layer. Finally the device is dried in $\mathrm{N}_{2}$ flow. The microscope photos in Fig. 10 show the device in some middle step.
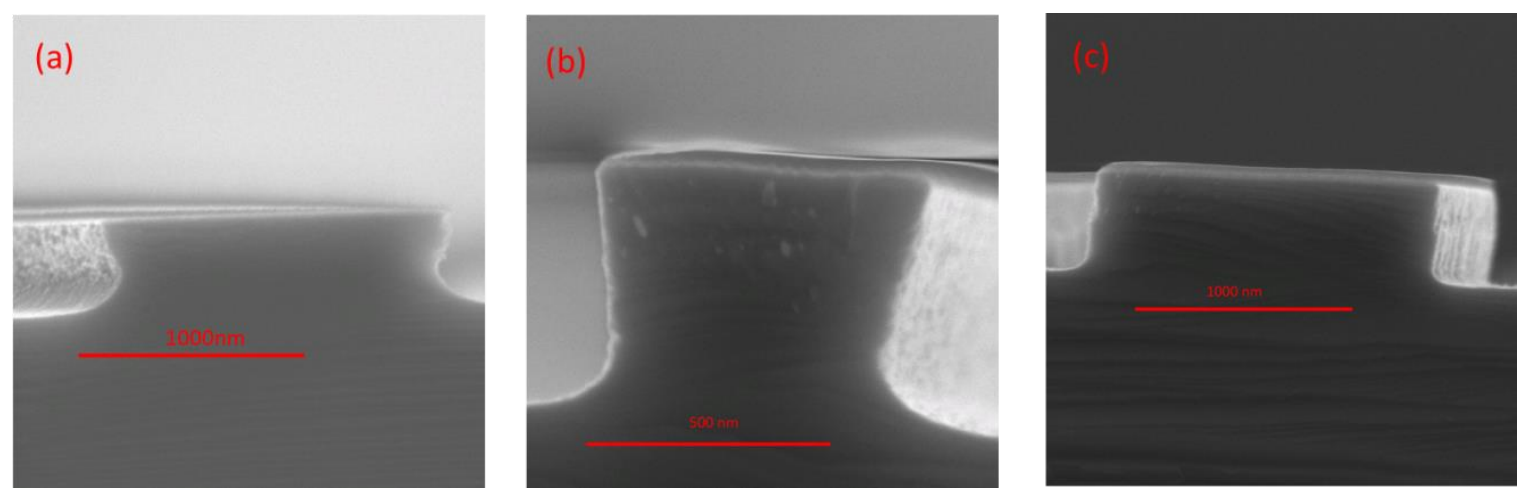

Fig. 9 The optimization of the RIE recipe to realize vertical side wall. (a) The etching result of the original recipe $\left(\mathrm{SF}_{6} 30 / \mathrm{O}_{2} 10 / \mathrm{CHF}_{3} 12\right)$ [24]; (b) The etching result of the improved recipe $\left(\mathrm{SF}_{6} 30 / \mathrm{O}_{2} 10 / \mathrm{CHF}_{3} 7\right)$; (c) The etching result of the final recipe $\left(\mathrm{SF}_{6} 30 / \mathrm{O}_{2} 14 / \mathrm{CHF}_{3} 12\right)$. 

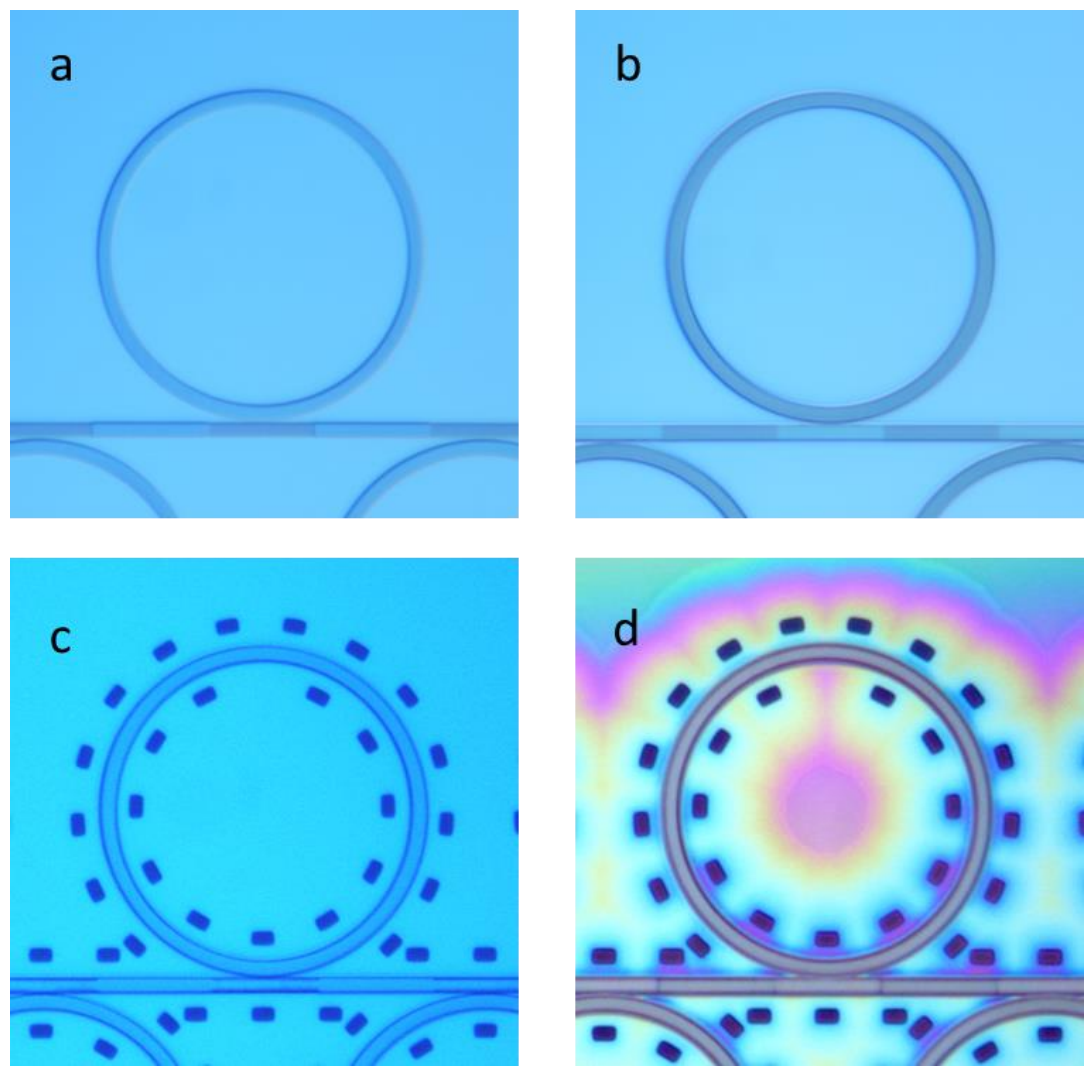

Fig. 10 The microscope photos of the device after (a) patterned resist for the waveguide and ring (b) transfering the pattern of waveguide and rings to the substrate by RIE (c) opening the holes on the substrate by RIE and (d) suspending the waveguide and ring 


\section{Chapter 3}

\section{Thermal tuning measurement}

\subsection{The principle of thermal tuning}

The transmission spectrum of a ring resonator is given by

$$
\operatorname{Tran}=1-\frac{\left(1-e^{-2 \gamma}\right)\left(1-t^{2}\right)}{\left(1-t e^{-\gamma}\right)^{2}+4 t e^{-\gamma} \sin ^{2}\left(\frac{\phi}{2}\right)}
$$

where $\gamma$ is the intrinsic loss efficient in the cavity, $t$ is the transmission coefficient of the coupling between the resonator and the bus waveguide, and $\phi$ is the phase change of the light wave after going around the cavity for one time. Generally speaking, $\phi$ is determined by the diameter of the $\operatorname{ring} D$, the wavelength of the light and the effective refractive index of the waveguide mode $n_{\text {eff }}$ in the following form

$$
\phi=\frac{2 \pi^{2} D}{\lambda} n_{\text {eff }}(\lambda, T) .
$$

Since $n_{\text {eff }}$ is dependent on the material index, which is also dependent on temperature, we can tune $\phi$ by changing temperature. When fixing wavelength and taking first order approximation with respect to $T$, Eq (3.2) becomes 


$$
\phi=\frac{2 \pi^{2} D}{\lambda}\left[n_{e f f}+\frac{\partial n_{e f f}}{\partial T}\left(T-T_{0}\right)\right]
$$

where $\partial n_{\text {eff }} / \partial T$ can be simulated by COMSOL. At the wavelength we're looking at, the themo-optic coefficient of $\mathrm{Si}$ is $1.7 \times 10^{-4} \mathrm{~K}^{-1}$ [25], which resulted in an effective index changing rate of $1.8 \times 10^{-4} \mathrm{~K}^{-1}$. The slightly larger value of $\partial \mathrm{n}_{\mathrm{eff}} / \partial \mathrm{T}$ is due to the fact that when increasing the refractive index of silicon, the optical mode will be more confined in Si core, which contribute a larger effective index. [26] In this way, we can get the information on $\gamma$ and $t$ by recording the thermal tuning spectrum $\operatorname{Tran}(T)$ and fitting it to Eqs. (3.1) and (3.3).

\subsection{Measurement setup for mid-IR U-shape waveguide}

The U-shape waveguide enables us to use only one lens for both input and output light collimation. [22] As indicated in Fig. 11, the output light from the chip propagates in a slightly different direction compared to the input light, thus can be separated and directed to the detector by a mirror put in a proper position and angle. [20] A PbSe-based IR detector is used to detect the mid-IR light. Since it only detect the $\mathrm{AC}$ component of the light, we use a chopper to modulate the light and pick up the frequency component corresponding to the modulation with a Lock-in amplifier locked to the chopper. The light is from a He-Ne laser with a fixed working wavelength at $3.4 \mu \mathrm{m}$. A thermoelectric cooler (TEC) and a temperature sensor connecting to a temperature controller are used to change and monitor the temperature of the chip. 


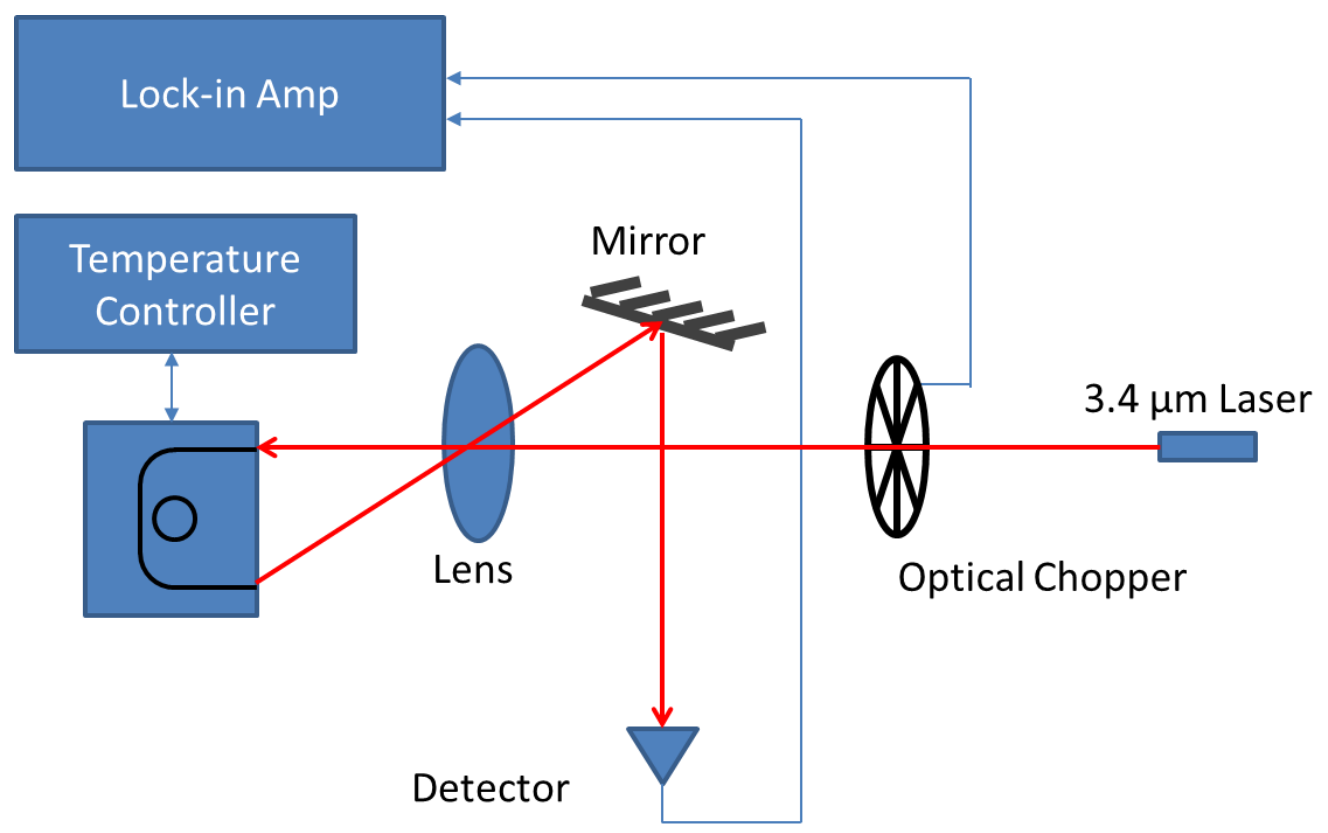

Fig. 11 Thermal tuning measuremnt system

\subsection{Measurement result}

A typical measurement result is shown as the green dots in Fig. 12, and is fitted as the blue curve. This is a SMW resonator with $\sim 35 \mu \mathrm{m}$ diameter and $1.5 \mu \mathrm{m}$ ridge width, and $\sim 360 \mathrm{~nm}$ waveguide-ring coupling gap. The fitting result indicates an intrinsic $Q$ of $\sim 11,000$, which corresponds to a waveguide loss of $\sim 27 \mathrm{~dB} / \mathrm{cm}$ [26]. 


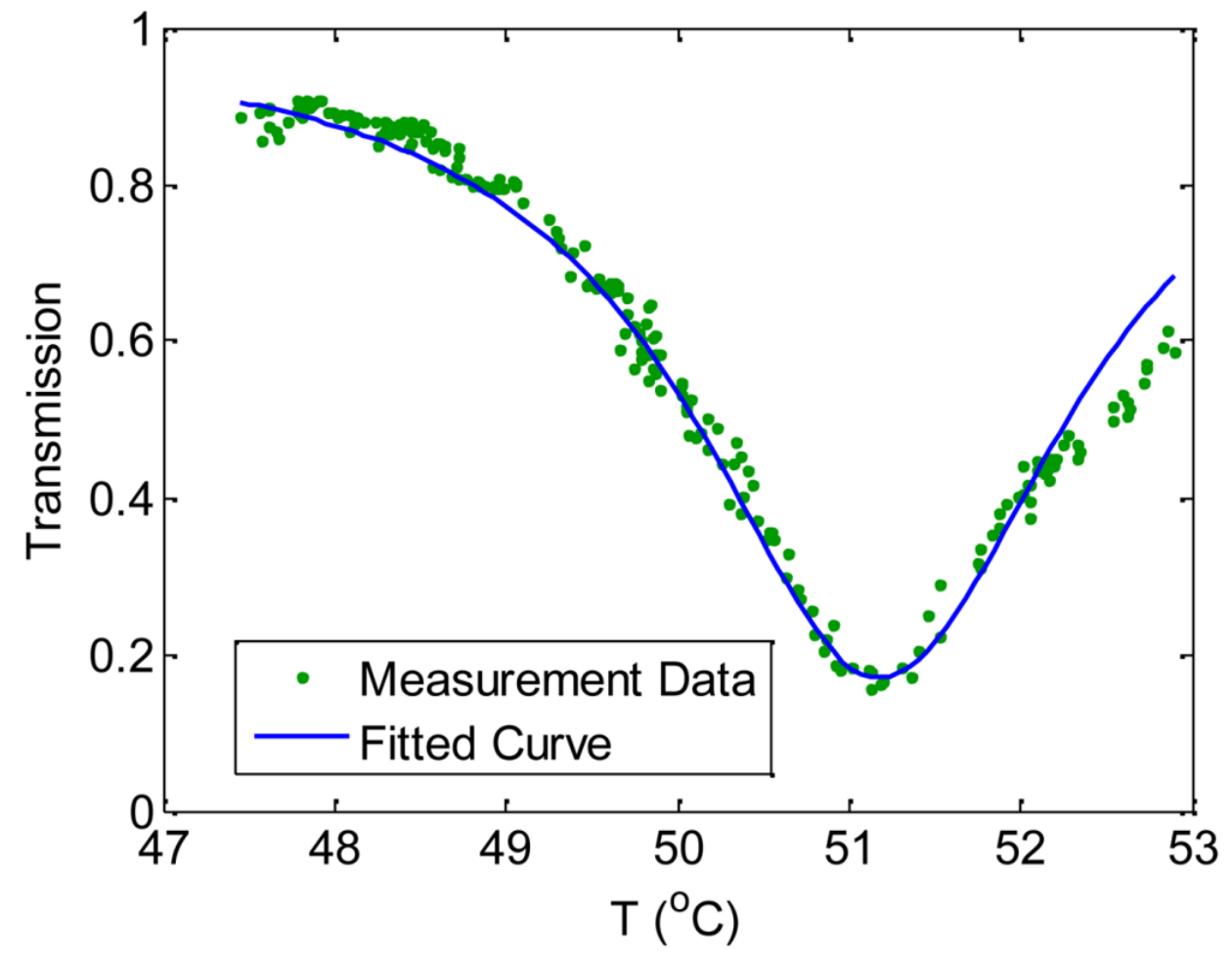

Fig. 12 A typical thermal tuning spectrum [20] 


\section{Chapter 4}

\section{Direct spectrum measurement by tunable QCL}

\subsection{Measurement setup}

The measurement setup is almost the same as Fig. 11, except that the laser is replaced by a tunable QCL. In our measurement, 2 QCLs are used successively, with mode-hoping free tunable ranges 5.116 - $5.221 \mu \mathrm{m}$ and $4.440-4.545 \mu \mathrm{m}$. Temperature control is also used to keep the chip at a fixed temperature to avoid thermal tuning from the environment temperature drifting.

\subsection{Transmission spectra of the SMW resonator}

A typical measurement result for the narrow-ridge resonators is presented in Fig. 13. The diameter of the ring is $\sim 35 \mu \mathrm{m}$ and the coupling gap is $\sim 900 \mathrm{~nm}$. The green dots is measured with an input power of $0.035 \mathrm{~mW}$, and is fitted with (3.1) and a first order approximation of (3.2) with fixed T and changing $\lambda$ as 


$$
\phi=\frac{2 \pi^{2} D}{\lambda}\left[n_{e f f}+\frac{\partial n_{e f f}}{\partial \lambda}\left(\lambda-\lambda_{0}\right)\right]
$$

The fitted intrinsic $Q$ of the resonator is $\sim 6,800$, corresponding to a waveguide loss of $\sim 31 \mathrm{~dB} / \mathrm{cm}$.

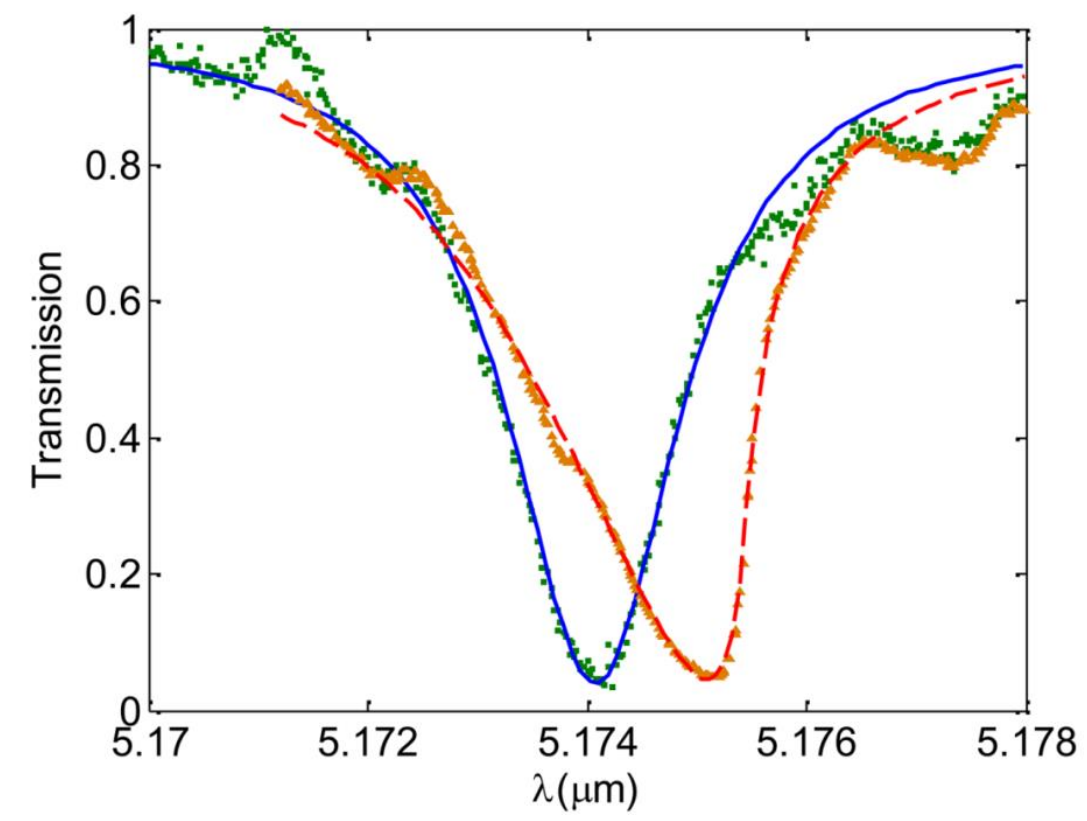

Fig. 13 Normalized transmission spectra of a narrow-ridge ring resonator [20]

When a high input power of $1.46 \mathrm{~mW}$ is applied to the device, a spectrum distortion can be seen clearly, as shown in orange triangles in Fig. 13. The change in the resonant wavelength is $1.2 \mathrm{~nm}$, which corresponds to a change in $\mathrm{n}_{\text {eff }}$ of $5.5 \times 10^{-4}$. Although Kerr effect can result in such phenomenon, the input power of $1.46 \mathrm{~mW}$ at this resonator only achieves refractive index change at the order of $10^{-7}$. [20] One the other hand, it only requires temperature change of $3{ }^{\circ} \mathrm{C}$ that leads to this change 
in $\mathrm{n}_{\mathrm{eff}}$ by thermal tuning. By COMSOL, we calculate that this temperature change only requires $0.18 \mathrm{~mW}$ heating power dissipated in the resonator area. Thus we believe the thermal effect can be the main reason.

To account for the phenomenon quantitatively, we add a term in (4.1) to represent the thermal tuning as

$$
\phi=\frac{2 \pi^{2} D}{\lambda}\left[n_{e f f}+\frac{\partial n_{e f f}}{\partial \lambda}\left(\lambda-\lambda_{0}\right)+\frac{\partial n_{e f f}}{\partial T} \frac{\eta P_{A}(1-T r a n)}{K}\right]
$$

where $P_{A}$ is the optical power arrived at the ring coupling zone (thus $P_{A}(1-\operatorname{Tran})$ is the power dissipation in the cavity), $\eta$ is ratio between the absorption loss and total loss and $K$ is the average temperature change in the cavity with unit heating power. Here $\partial n_{\text {eff }} / \partial T$ and $K$ is simulated by COMSOL (the simulation model is shown in ), and $\eta$ is a fitting parameter. By fitting the measured spectra with Eqs. (3.1) and (4.2), which is shown in Fig. 13 as the red dashed line, $\eta$ is found to be $\sim 0.21$. Therefore, optical absorption accounts for a small portion of the waveguide loss at $\sim 6.5 \mathrm{~dB} / \mathrm{cm}$, while scattering accounts for the rest. [20] 


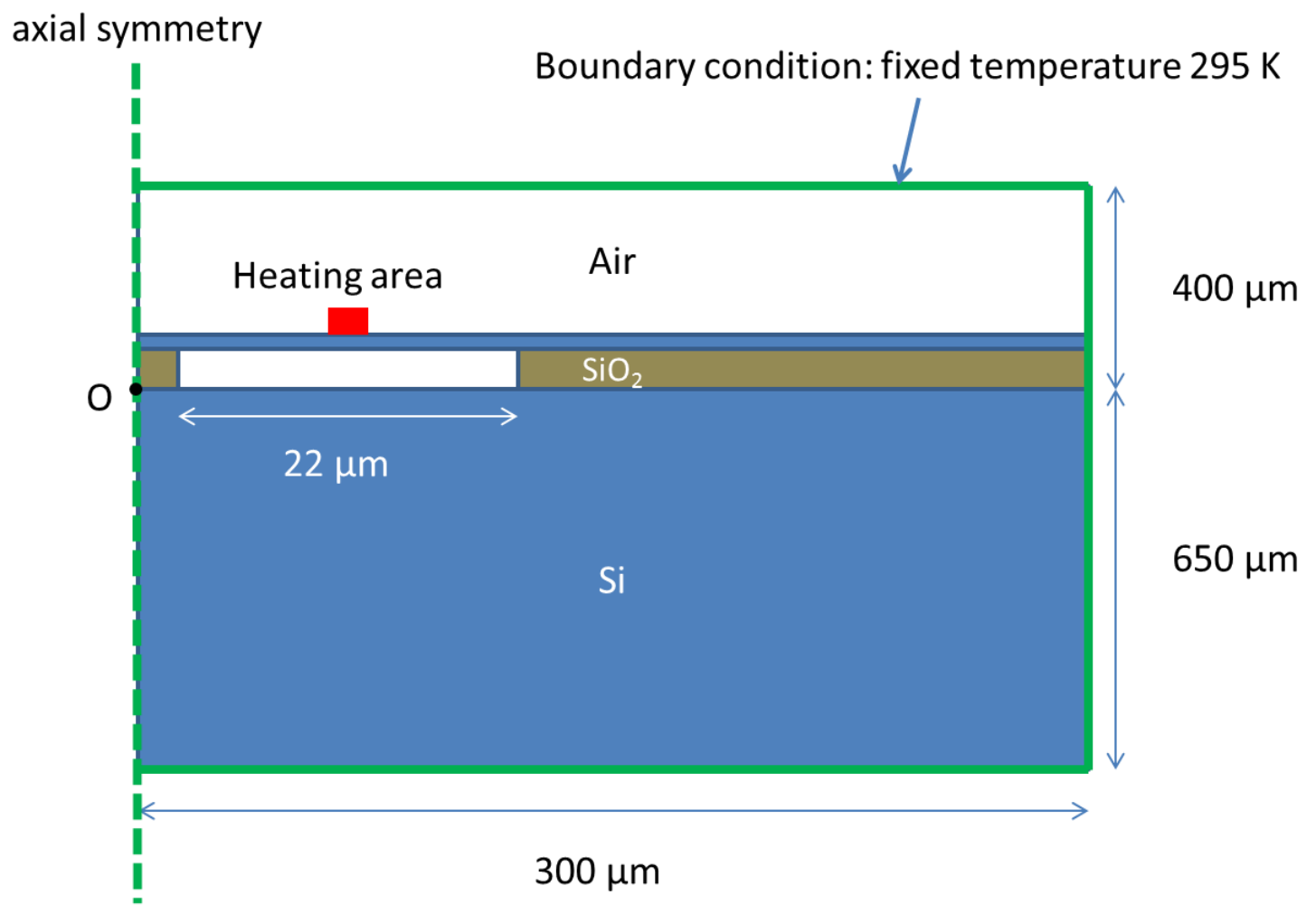

Fig. 14 The COMSOL model for the simulation. Axial symmetry condition is applied on the left edge. The temperature of all four edges is fixed at $295 \mathrm{~K}$. The initial temperature of all parts is also $295 \mathrm{~K}$. The heating is assumed to be homogenouse at the ridge area. The ridge is $1.5 \mu \mathrm{m}$ wide. The height of the ridge and the thickness of the slab are $0.5 \mu \mathrm{m}$ and $0.25 \mu \mathrm{m}$ respectively.

The transmission spectrum of the same device is also measured near $4.5 \mu \mathrm{m}$ wavelength and is shown as the red dots and fitted as the blue curve in Fig. 15. The fitting result indicates an intrinsic $Q$ of 16,000 with a loss coefficient of $15 \mathrm{~dB} / \mathrm{cm}$. The reduction in transmission loss is due to the weaker field at the surface of the waveguide in shortening wavelength, which confirms that the loss is on the surface of the device, and should be able to decrease by improving fabrication procedures. 


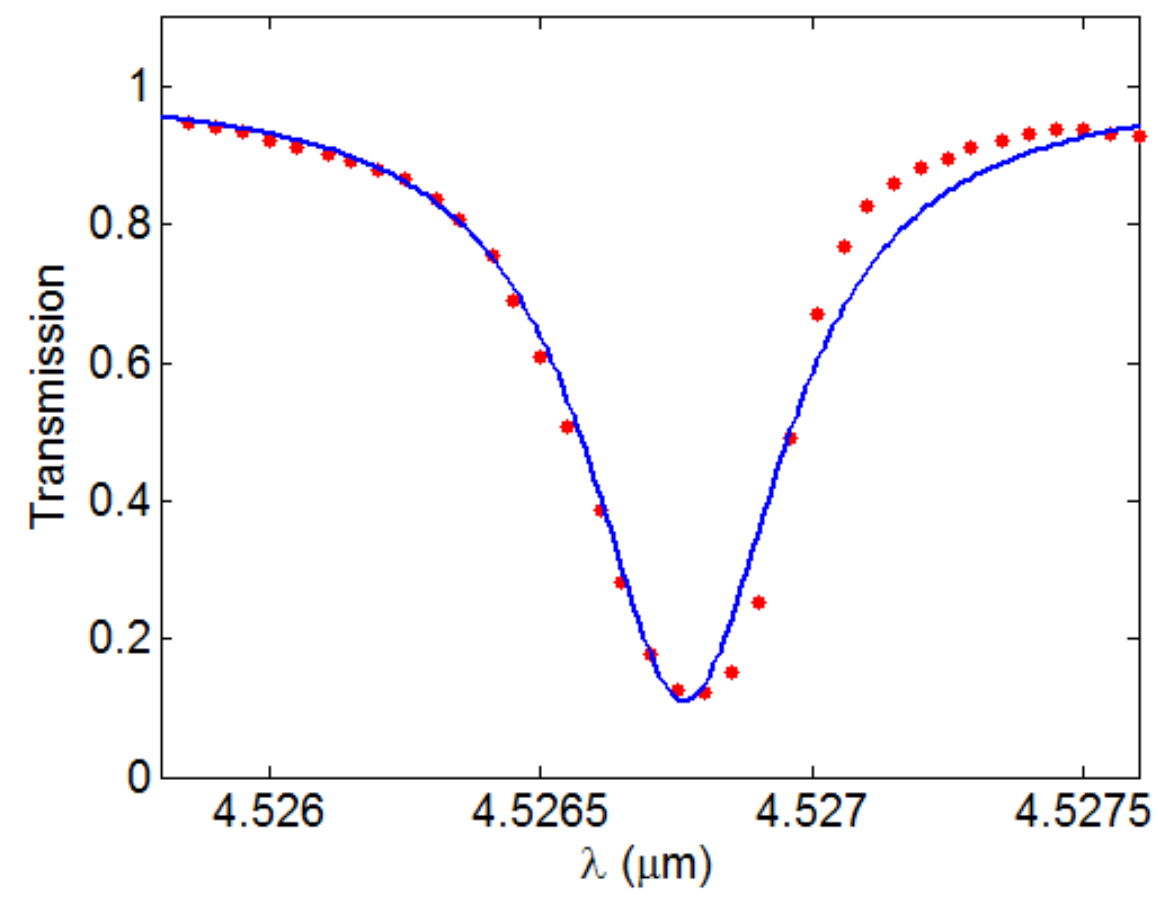

Fig. 15 Transmission spectrum of the SMW resonator near $4.5 \mu \mathrm{m}$.

\subsection{Transmission spectra of MMW resonator}

A typical spectrum for the wide-ridge resonator (waveguide diameter is designed at $3 \mu \mathrm{m}$ ) is measured as the red dots in Fig. 16, and fitted as the blue curve. The diameter of the ring is $\sim 60 \mu \mathrm{m}$ and the ring-waveguide coupling gap is designed at $0.44 \mu \mathrm{m}$, and the measurement is conducted with a low input power of $0.044 \mathrm{~mW}$. The loaded $Q$ is $\sim 21,000$, while the intrinsic $Q$ is as high as 35,000 , which corresponds to a loss coefficient as low as $6.5 \mathrm{~dB} / \mathrm{cm}$. 


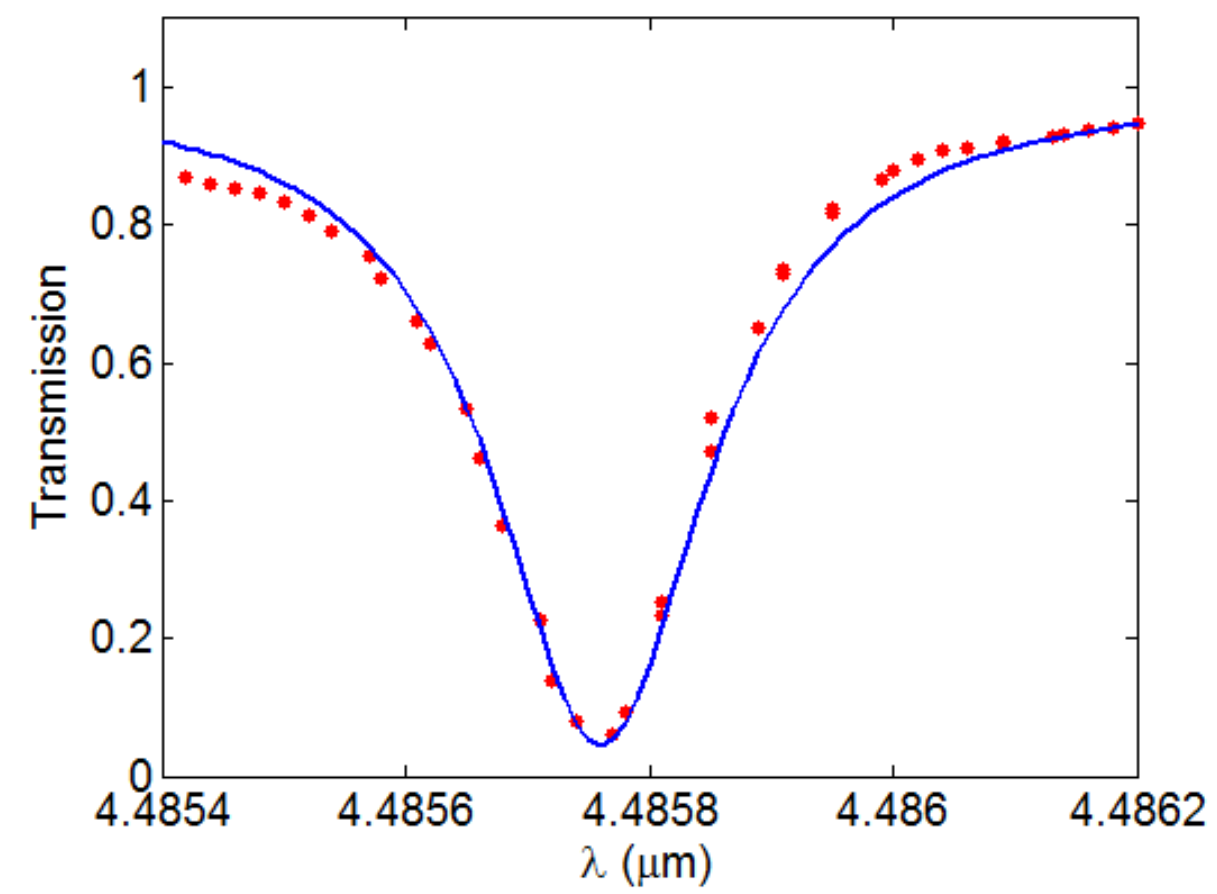

Fig. 16 The transmission spectrum and fitting result for a wide-ridge resonator.

It is clear that when the dimension of the waveguide becomes large, the optical loss reduced significantly. This is also consistent with the assumption that the light power is mainly dissipated at the surface of the waveguide, since in such a case, when the dimension of the waveguide becomes larger, better confinement is achieved and less power is absorbed and scattered at the surface of the waveguide. [27] 


\subsection{Thermal tuning rate measurement}

We experimentally measured the thermal tuning rate, as indicated in Fig. 17. The green squares are measured at $22{ }^{\circ} \mathrm{C}$ and fitted in the blue curve, while the orange squares are measured at $27^{\circ} \mathrm{C}$ and fitted in the red curve. The temperature change of $5{ }^{\circ} \mathrm{C}$ gives a change of $1.03 \mathrm{~nm}$ in resonant wavelength, corresponding to a thermal tuning rate of $0.21 \mathrm{~nm} /{ }^{\circ} \mathrm{C}$, which is the same as estimated from our simulation result and the equation below

$$
\Delta \lambda=\frac{\lambda}{n_{g}} \frac{\partial n_{e f f}}{\partial T} \Delta T
$$

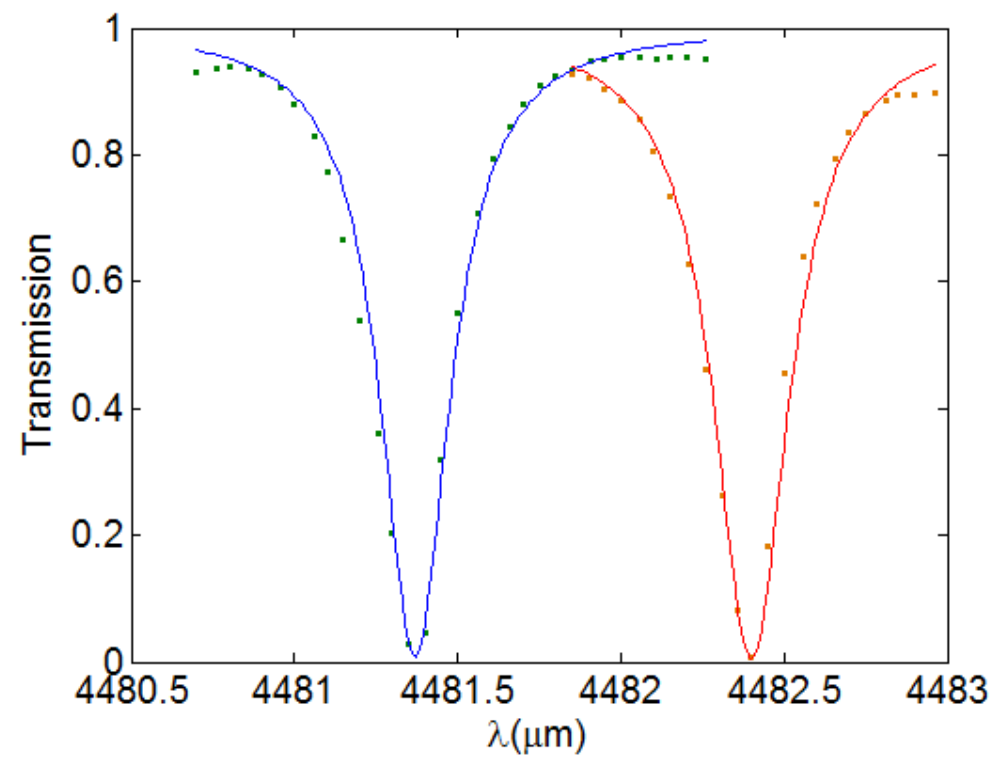

Fig. 17 Thermal tuning rate measurement. The green squares are measured at $22{ }^{\circ} \mathrm{C}$ and fitted in the blue curve, while the orange squares are measured at $27^{\circ} \mathrm{C}$ and fitted in the red curve. 


\section{Chapter 5}

\section{All-optical modulation}

\subsection{Measurement system}

Absorption induced temperature change enable us to perform all-optical thermo-optical switching at the mid-infrared range. The measurement system is shown in Fig. 18. The intense light from the QCL is collimated with the probe light from the He-Ne laser, and coupled to the device simultaneously. After coupling out of the chip, the two beams are separated by a grating, thus the detector only measures the probe light. The pump light is modulated at a curtain frequency $f$ by current modulation. The thermal tuning curve of the cavity transduces the oscillation in temperature to oscillation in output probe light, as indicated in Fig. 19. Locked to the modulation signal, the measurement system only picks up the fcomponent from the probe light. The temperature of the chip is maintained at $50{ }^{\circ} \mathrm{C}$ to maximize the modulation depth, which happens when the device is biased at the point with the largest slope in the thermo-optical spectrum (see Fig. 12). 


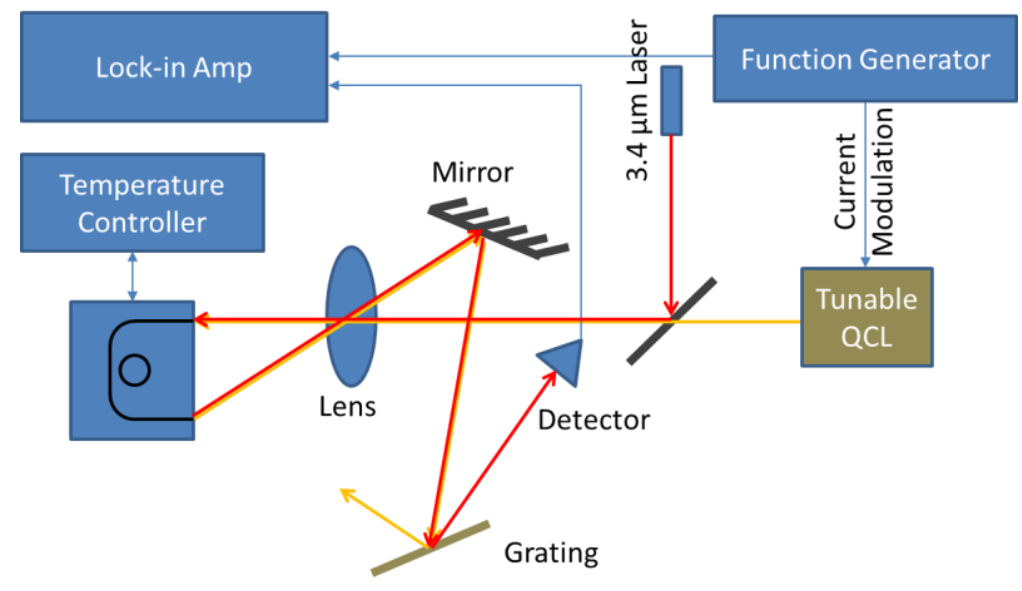

Fig. 18 All-optical thermo-optical modulation measurment system.

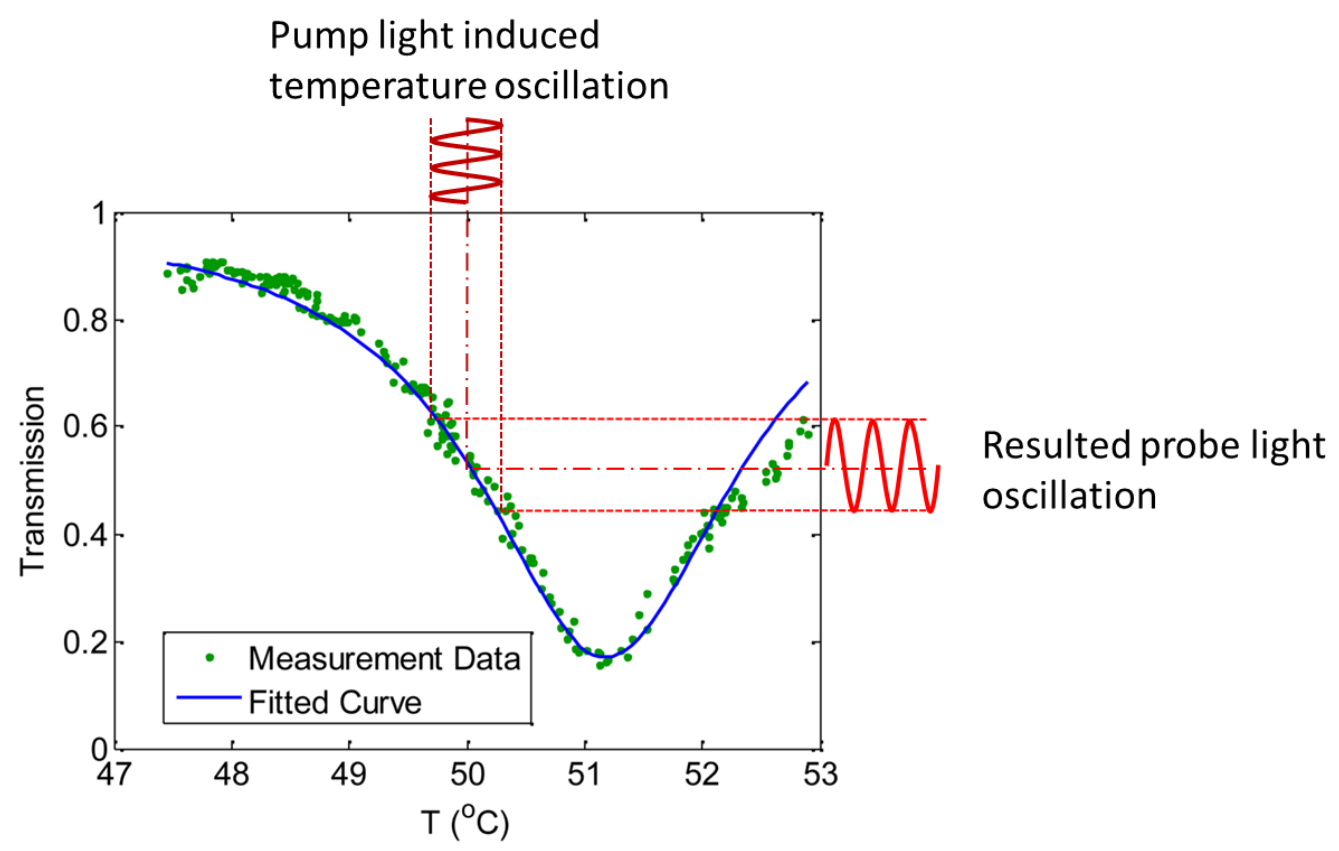

Fig. 19 The principle of all-optical thermal-optical modulation. 


\subsection{All-optical modulation results}

Since the modulation is mainly caused by the thermal dissipation of the pump light in the cavity, the response should also reflect the transmission spectrum of the pump light. This is indeed the case we find in Fig. 20. The modulation strength $\mathrm{M}$ is maximized when the pump light wavelength is aligned with the resonance wavelength of the cavity. [20] When the pump light is tuned away from the resonant wavelength, heating effect from the waveguide near-by contributes to the modulation effect, making a non-zero background. The loaded quality factor of this device for the $5.2 \mu \mathrm{m}$ pump light is quite low, since the coupling gap of this device is only $\sim 360 \mathrm{~nm}$. The fitting result gives an intrinsic $\mathrm{Q}$ of 6,400 , corresponding to a loss coefficient of $32 \mathrm{~dB} / \mathrm{cm}$, which is close to the conclusion of Fig. 13 .

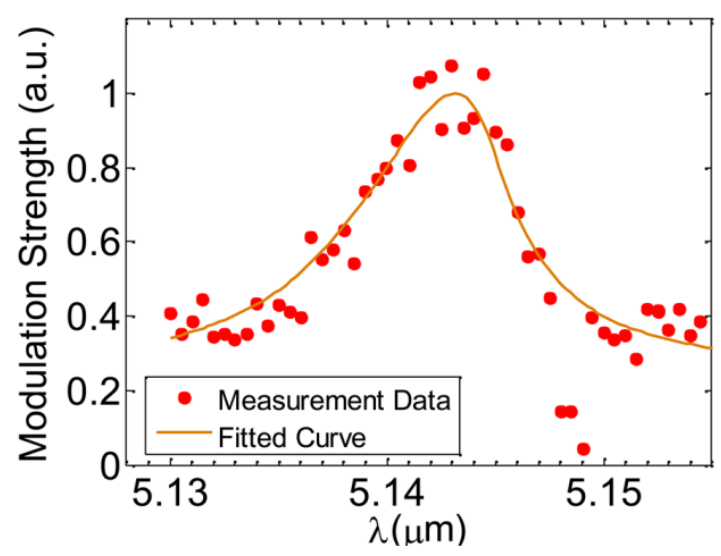

Fig. 20 Optical modulation response with respect to pump wavelength. 
To estimate the bandwidth of this modulation and characterize the thermal response time, we measure the modulation strength at different modulation frequencies. To exclude the effect from the measurement system, the result is normalized to the pump light signal measured afterwards. The measurement result is shown as the purple stars in Fig. 21. It is fitted with a low-pass filter model (orange dashed line) with a thermal response time of $7.5 \mu$ s. The relatively long response time confirm our speculation that the modulation is due to a slow process such as thermal effect. [20] A time domain simulation by COMSOL gives the frequency response as the red curve in Fig. 21. It has a slightly larger bandwidth, which we believe due to neglecting the bus waveguide near the ring, resulting in a smaller heat capacitance and faster response.

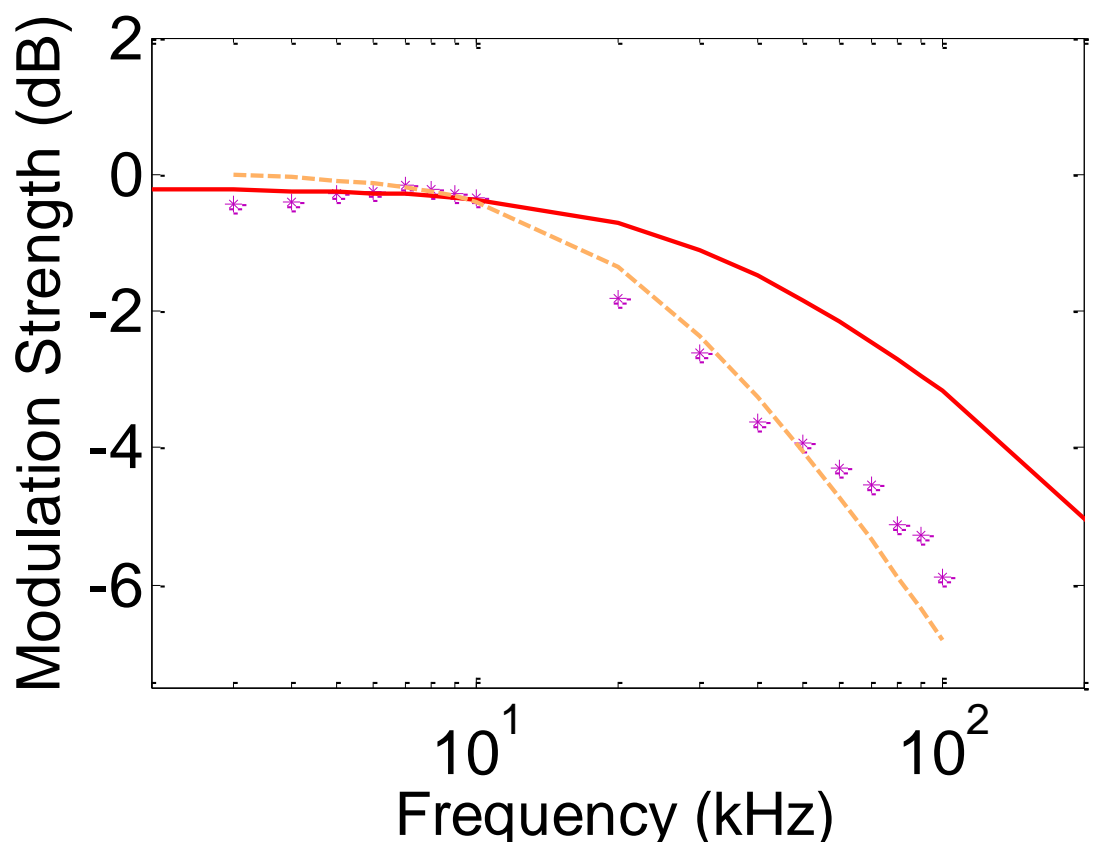

Fig. 21 Bandwidth measurement of the thermo-optical modulation [20] 


\section{Chapter 6}

\section{On-chip electrical heater}

To better understand the thermal tuning strength of the suspended structure and realize better thermal control of the resonance, on-chip electrical heater is designed for our device. To avoid disturbance to the resonator, resistive heater is designed outside of the outer holes of the resonator waveguide, as presented in Fig. 22. The width of each metal line is $1.5 \mu \mathrm{m}$, and the gap between them is also $1.5 \mu \mathrm{m}$. The distance between the inner line and the waveguide is $6 \mu \mathrm{m}$. $\mathrm{Cr}$ is used as the heater material, due to its relatively high resistance and resist to BHF etch [28], which enables us to fabricate the heater before suspending the structure. From a simulation by COMSOL, the heating efficiency is $1^{\circ} \mathrm{C} / \mathrm{mA}$. 


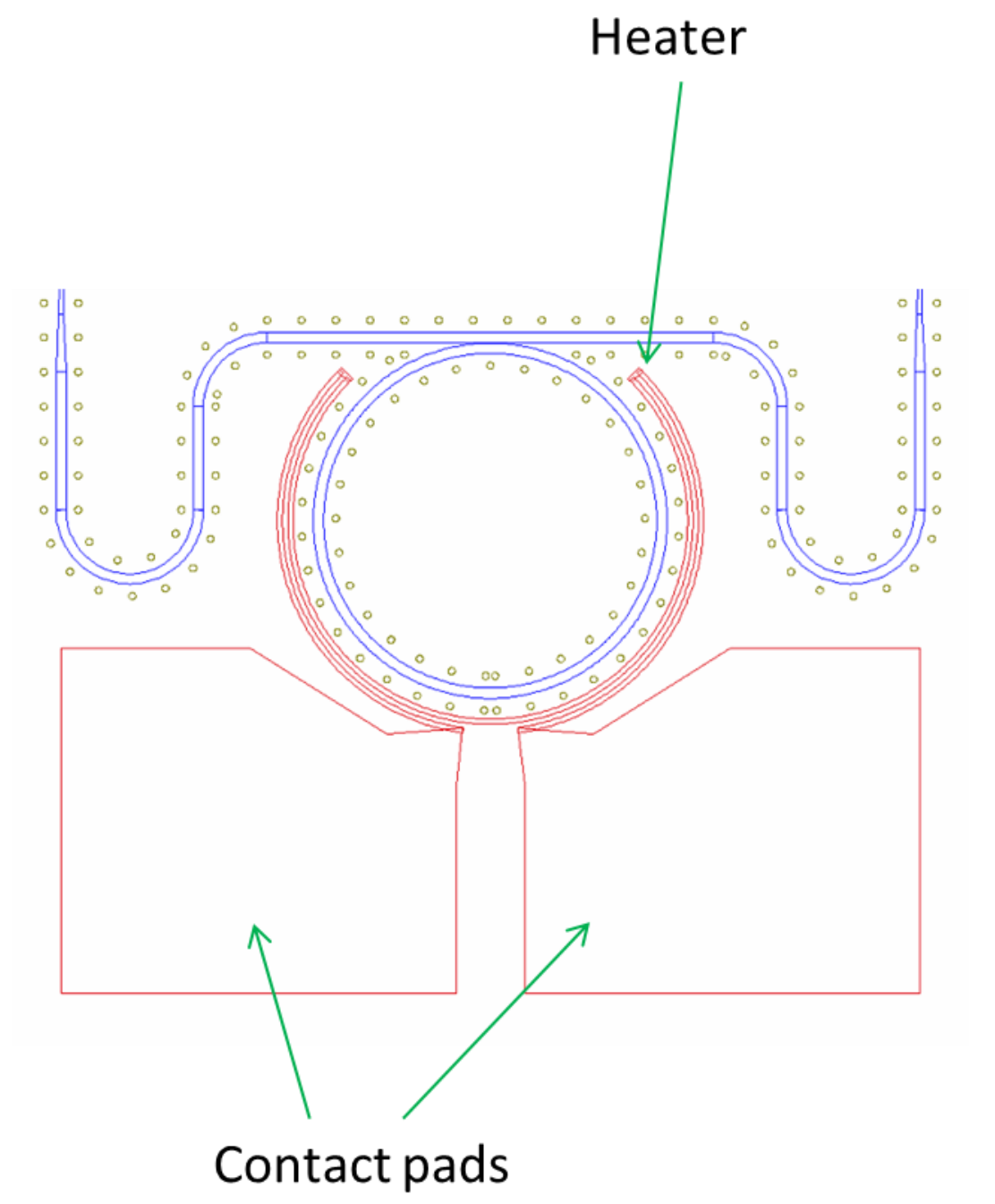

Fig. 22 On-chip electrical heater design. The heater and electrodes are shown as the area surrounded by red curves.

An EBL is done to check the feasibility of the pattern design. The challenge is using quite large current and aperture for the SEM. It turns out that such configuration works well (as shown in Fig. 23), which get us rid of making optical lithography. 


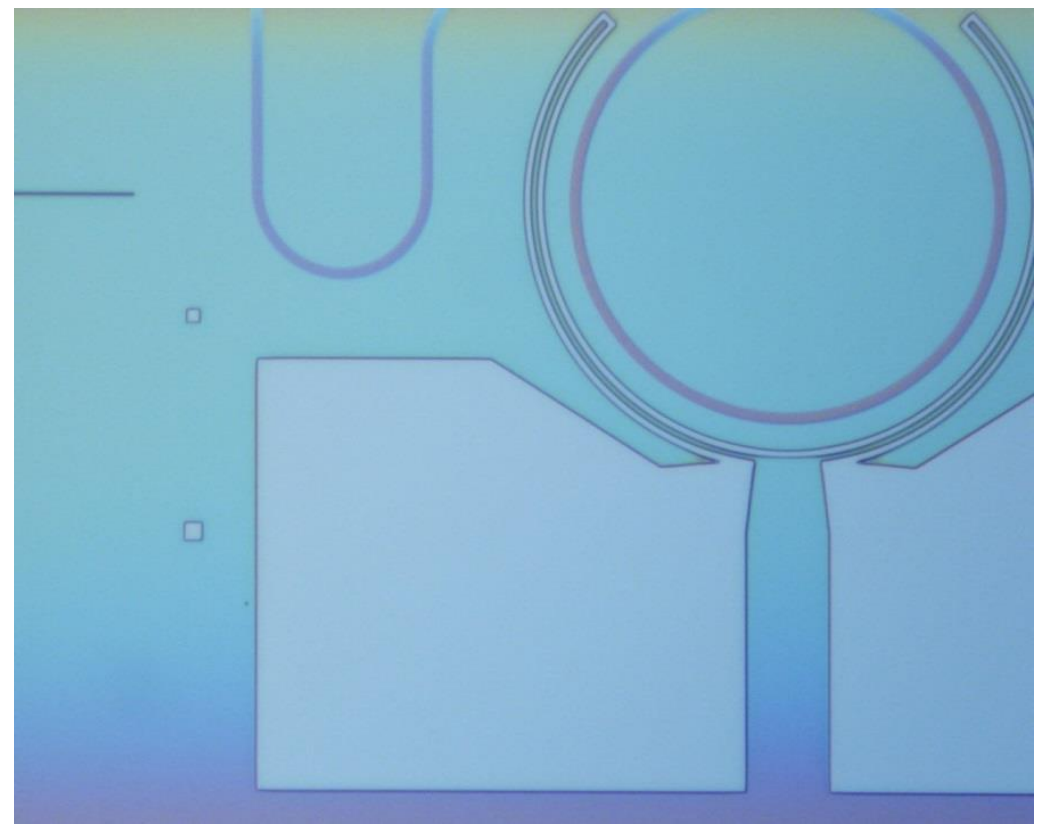

Fig. 23 Lithography result of the heater 


\section{Chapter 7}

\section{Summery}

In this thesis, suspended silicon waveguide and ring resonator working in mid-infrared range are fabricated and characterized. The SMW resonator gives intrinsic $Q$ of 6,800 and exhibits loss of $\sim 31 \mathrm{~dB} / \mathrm{cm}$ when measured near $5.2 \mu \mathrm{m}$ wavelength, while the intrinsic $Q$ increases to 16,000 and the loss decreases to 15 $\mathrm{dB} / \mathrm{cm}$ when measured near $4.5 \mu \mathrm{m}$. The resonator by the multimode waveguide presents intrinsic $Q$ of 35,000 while the loss coefficient is $6.5 \mathrm{~dB} / \mathrm{cm}$. Spectrum distortion is modeled by light absorption induced heating, and $\sim 20 \%$ optical power loss contribute to this effect. All-optical thermo-optical switching is demonstrated, and the response time is measured to be $7.5 \mu$ s. On-chip electrical heater is designed. Preliminary experiment suggests it is possible to use our SEM-based EBL system to pattern the heater and electrodes.

The optical loss mainly occurs on the surface of the waveguide, thus can be reduced by improving fabrication procedures. The etching depth is at the upper limit for the resist and etching process we use. Better result should be obtained if 
we introduce metal mask, which guarantees a much larger selectivity. Postprocesses such as piranha washing [4] and oxidation smoothing [29] can also be used to clean and smooth the surface, and further reduce the optical loss. 


\section{References}

1. K. Miyamoto, K. Ishibashi, K. Hiroi, Y. Kimura, H. Ishii, and M. Niwano, "Labelfree detection and classification of DNA by surface vibration spectroscopy in conjugation with electrophoresis," Appl. Phys. Lett. 86, 053902-053903 (2005).

2. F. K. Tittel, Y. Bakhirkin, A. Kosterev, R. Lewicki, S. So, G. Wysocki, and R. F. Curl, "Recent advances and applications of mid-infrared based trace gas sensor technology," Proc. SPIE 6900, 69000Z (2008).

3. R. A. Soref, S. J. Emelett, and W. R. Buchwald, "Silicon waveguided components for the long-wave infrared region," Journal of Optics A: Pure and Applied Optics 8, 840 (2006).

4. T. Baehr-Jones, A. Spott, R. Ilic, A. Spott, B. Penkov, W. Asher, and M. Hochberg, "Silicon-on-sapphire integrated waveguides for the mid-infrared," Opt. Express 18, 12127-12135 (2010).

5. T. J. Kippenberg, R. Holzwarth, and S. A. Diddams, "Microresonator-Based Optical Frequency Combs," Science 332, 555-559 (2011).

6. Z. Cheng, X. Chen, C. Y. Wong, K. Xu, C. K. Y. Fung, Y. M. Chen, and H. K. Tsang, "Focusing subwavelength grating coupler for mid-infrared suspended membrane waveguide," Opt. Lett. 37, 1217-1219 (2012).

7. Z. Cheng, X. Chen, C. Y. Wong, K. Xu, and H. K. Tsang, "Mid-infrared Suspended Membrane Waveguide and Ring Resonator on Silicon-onInsulator," IEEE Photon. J. 4, 1510-1519 (2012).

8. A. Schliesser, N. Picque, and T. W. Hansch, "Mid-infrared frequency combs," Nature Photonics 6, 440-449 (2012).

9. R. Soref, "Mid-infrared photonics in silicon and germanium," Nat. Photon. 4, 495-497 (2010).

10. X. Liu, R. M. Osgood, Y. A. Vlasov, and M. J. GreenWilliam, "Mid-infrared optical parametric amplifier using silicon nanophotonic waveguides," Nat Photon 4, 557-560 (2010).

11. S. Zlatanovic, J. S. Park, S. Moro, J. M. C. Boggio, I. B. Divliansky, AlicNikola, S. Mookherjea, and S. Radic, "Mid-infrared wavelength conversion in silicon waveguides using ultracompact telecom-band-derived pump source," Nat Photon 4, 561-564 (2010).

12. X. Liu, B. Kuyken, G. Roelkens, R. Baets, R. M. Osgood, and W. M. J. Green, "Bridging the mid-infrared-to-telecom gap with silicon nanophotonic spectral translation," Nat Photon 6, 667-671 (2012).

13. S. A. Diddams, T. Udem, J. C. Bergquist, E. A. Curtis, R. E. Drullinger, L. Hollberg, W. M. Itano, W. D. Lee, C. W. Oates, K. R. Vogel, and D. J. Wineland, "An Optical Clock Based on a Single Trapped 199Hg+ Ion," Science 293, 825828 (2001). 
14. A. Shelkovnikov, R. J. Butcher, C. Chardonnet, and A. Amy-Klein, "Stability of the Proton-to-Electron Mass Ratio," Physical Review Letters 100, 150801 (2008).

15. M. T. Murphy, T. Udem, R. Holzwarth, A. Sizmann, L. Pasquini, C. AraujoHauck, H. Dekker, S. D'Odorico, M. Fischer, T. W. Hansch, and A. Manescau, "High-precision wavelength calibration of astronomical spectrographs with laser frequency combs," Mon Not R Astron Soc 380, 839-847 (2007).

16. F. Keilmann, C. Gohle, and R. Holzwarth, "Time-domain mid-infrared frequency-comb spectrometer," Opt. Lett. 29, 1542-1544 (2004).

17. P. Del/'Haye, A. Schliesser, O. Arcizet, T. Wilken, R. Holzwarth, and T. J. Kippenberg, "Optical frequency comb generation from a monolithic microresonator," Nature 450, 1214-1217 (2007).

18. T. J. Kippenberg, S. M. Spillane, and K. J. Vahala, "Kerr-nonlinearity optical parametric oscillation in an ultrahigh-Q toroid microcavity," Physical Review Letters 93(2004).

19. J. S. Levy, A. Gondarenko, M. A. Foster, A. C. Turner-Foster, A. L. Gaeta, and M. Lipson, "CMOS-compatible multiple-wavelength oscillator for on-chip optical interconnects," Nat Photon 4, 37-40 (2010).

20. Y. Xia, C. Qiu, X. Zhang, W. Gao, J. Shu, and Q. Xu, "Suspended Si ring resonator for mid-IR application," Opt. Lett. 38, 1122-1124 (2013).

21. A. S. Richard, J. E. Stephen, and R. B. Walter, "Silicon waveguided components for the long-wave infrared region," Journal of Optics A: Pure and Applied Optics 8, 840 (2006).

22. Q. Xu, D. Fattal, and R. G. Beausoleil, "Silicon microring resonators with 1.5$\mu \mathrm{m}$ radius," Opt. Express 16, 4309-4315 (2008).

23. A. B. Matsko, A. A. Savchenkov, and L. Maleki, "Normal group-velocity dispersion Kerr frequency comb," Opt. Lett. 37, 43-45 (2012).

24. R. Legtenberg, H. Jansen, M. Deboer, and M. Elwenspoek, "Anisotropic Reactive Ion Etching of Silicon Using $\mathrm{SF}_{6} / \mathrm{O}_{2} / \mathrm{CHF}_{3}$ Gas Mixtures," Journal of The Electrochemical Society 142, 2020-2028 (1995).

25. B. J. Frey, D. B. Leviton, and T. J. Madison, "Temperature-dependent refractive index of silicon and germanium," Proc. SPIE 6273, 62732J (2006).

26. C. Y. Wong, Z. Cheng, X. Chen, K. Xu, C. K. Y. Fung, Y. M. Chen, and H. K. Tsang, "Characterization of Mid-Infrared Silicon-on-Sapphire Microring Resonators With Thermal Tuning," IEEE Photon. J. 4, 1095-1102 (2012).

27. K. K. Lee, D. R. Lim, H.-C. Luan, A. Agarwal, J. Foresi, and L. C. Kimerling, "Effect of size and roughness on light transmission in a $\mathrm{Si} / \mathrm{SiO}$ [sub 2] waveguide: Experiments and model," Applied Physics Letters 77, 1617-1619 (2000).

28. K. R. Williams, K. Gupta, and M. Wasilik, "Etch rates for micromachining processing - Part II," Journal of Microelectromechanical Systems 12, 761-778 (2003). 
29. D. K. Sparacin, S. J. Spector, and L. C. Kimerling, "Silicon waveguide sidewall smoothing by wet chemical oxidation," J. Lightwave Technol. 23, 2455-2461 (2005). 